\title{
Robust Feed-back Control of Distributed Chemical Reaction Systems
}

\author{
Carlos Vilas, Míriam R. García, Julio R. Banga, \\ Antonio A. Alonso * \\ Process Engineering Group, IIM-CSIC, Eduardo Cabello 6, 36208 Vigo, Spain.
}

\begin{abstract}
There are many distributed processes in the chemical industry as it is the case of tubular reactors in which the parameters or the structure of the reaction terms are only a rough approximation of reality. In order to efficiently control this kind of systems, it is important to take into account this lack of detailed information (robustness). In this work, we make use of the classical theory on the robust nonlinear control for finite dimensional systems and extend it to distributed process systems by taking advantage of the special nature of dissipative systems. In this way, theoretical issues related to the nonlinearity of the diffusion terms and inhomogeneous boundary conditions are handled by means of the Kirchhoff and state transformations, respectively. In addition, and for practical reasons, the problem of controller saturation is considered. The different aspects of the methodology will be illustrated through a number of computational experiments concerning non-isothermal tubular reactors with convection and/or diffusion terms.
\end{abstract}

Key words: Reaction-Diffusion-Convection, Dissipative Systems, Tubular Reactors, Distributed Process Systems, Robust Nonlinear Control, PDE.

\section{Introduction}

Over the recent years, a considerable research effort concentrated on the design of control policies for distributed process systems (Christofides, 2001). Standard approaches to the control of this kind of systems are based on the

*

Email addresses: antonio@iim.csic.es, Phone: +34986231930 Ext. 251

(Antonio A. Alonso ).

URL: http://www.iim.csic.es/ gingproc/ (Antonio A. Alonso). 
spatial discretization of the original set of partial differential equations (PDEs) to obtain a set of ordinary differential equations (ODEs). This allows us to employ standard finite-dimensional methods to construct the controller (Dochain et al., 1992; Gundepudi and Friedly, 1998). Nevertheless, there exist several disadvantages in these methods. For instance, the controllability and observability properties would depend on the number of discretization points as well as its location. Moreover, in processes where the distribution is strong, these approaches may lead to a poor control quality (Christofides, 2001). In addition, the resulting set of ODEs is computationally involved due to its high dimensionality which could make the approach unsuitable for real time applications.

Due to these disadvantages, new methods based on spectral decomposition techniques, which take into account the spatially distributed nature of these systems, have emerged. This approach takes advantage of the spatial differential operator structure and uses the Galerkin method to approximate the system by a low-dimensional set of ODEs and to design the controller (Shvartsman and Kevrekidis, 1998). Christofides and coworkers -see, for example (Christofides and Daoutidis, 1996; Shi et al., 2006)- employed this approach to derive stabilizing controllers based on feed-back linearization and applied it to chemical systems such as tubular reactors or particulate processes, among others. This methodology has been widely employed by many authors in the context of control of chemical reactors -see (Hoo and Zheng, 2001; Alonso et al., 2004b) and references therein- or regarding biological systems (Smagina et al., 2002; Vilas et al., 2006).

On the other hand, in chemical and biological processes, detailed information on the structure of nonlinear terms or system parameters is frequently not available, calling for the development of robust controllers. Regarding this problem, different approaches have been recently considered. The Galerkin projection, mentioned above, was also employed in this context for the synthesis of robust controllers and optimal sensor placement (Antoniades and Christofides, 2002; Demetriou and Kazantzis, 2005). From another point of view, in Ydstie and Alonso (1997); Alonso and Ydstie (2001); Alonso et al. (2002b) the authors made use of the second law of thermodynamics to design a simple robust control structure for the stabilization of RD systems. This formalism was applied in Alonso et al. (2004a) to explore connections between physics of dissipative systems and nonlinear robust control. The results of this paper were extended in Vilas et al. (2006) to the robust stabilization of complex behaviors such as limit cycles in reaction-diffusion (RD) systems.

At this point, it must be remarked that the nonlinearity in reaction-diffusionconvection (RDC) systems may appear in both the reaction and field dependent diffusivity terms. Phase change processes, among others, fit into the second type of nonlinearity. Due to the special structure of these systems, clas- 
sical numerical methods usually result prohibitive. The Kirchhoff transform (Saro et al., 1995) allows us to reformulate the problem in such a way that the field dependent diffusivity is removed from the equations. This methodology was successfully applied in different fields such as freezing or thawing of foods with arbitrary 3D geometries (Scheerlinck et al., 2001), melting processes or thermal processes induced by laser irradiation (Conde et al., 2005), among others. The advantages of using this transformation are not only numerical but theoretical as well since the structure of the reformulated problem allows us to extract interesting properties of this kind of systems for control and stability analysis purposes (Alonso et al., 2004a).

In this work, we deal with the tracking control problem of a class of distributed chemical reaction systems when structural and/or parametric model uncertainties are present. To that purpose, we extend the methodology developed in Alonso et al. (2004a) and Vilas et al. (2006) to RDC systems. In this regard, the possibility of using the Kirchhoff transform is explored and the stability properties of the control law are proved by taking advantage of the structure of the transformed system. In addition, the boundary transformations proposed by Balsa-Canto et al. (2004), are employed so as to deal with problems with inhomogeneous boundary conditions. Finally, and for practical considerations, some issues related to the saturation of the controllers are discussed. The different aspects of the methodology are illustrated through a number of case studies concerning chemical tubular reactors.

The paper is organized as follows: First, in Section 2 a general description of RDC systems is presented. In Section 3, the basis for the derivation of low dimensional approximations to the PDE system are set up. In the next section, it is shown that such an approximation is always possible to find in the class of processes considered. After this, in Section 5, the control laws are derived by taking advantage of the previously developed theory. The results are then applied to a number of case studies in Section 6. Finally, the article concludes with some comments on the theory and the results obtained in the computational experiments.

\section{Description of Reaction-Diffusion-Convection System}

In describing the class of systems we are dealing with, we will follow the methodology employed by Alonso and coworkers (Alonso and Ydstie, 2001; Alonso et al., 2004a) and represent the dynamic evolution of a process system by a set of inventory balances which lead to a set of partial differential equations of the form:

$$
\frac{\partial z}{\partial t}+\nabla X=\Sigma(z)+p
$$


where $z=\left[z_{1}, z_{2}, \ldots, z_{n s}\right]^{T}$ is the vector of internal energy and concentration density of chemical species (the field), with $n s$ being the number of states. $\Sigma(z)$ represents the production terms and $p$ stands for the control. The vector function $X$ collects the microscopic fluxes through the domain and it can be partitioned into its convective and diffusive contributions, as follows:

$$
X=\mathbf{v} z-L(A) \nabla A,
$$

with $\mathbf{v}$ being the fluid velocity field. $L(\cdot)$ represents a positive definite matrix containing the diffusion coefficients. The set of intensive variables $A=$ $\left[A_{1}, A_{2}, \ldots, A_{n s}\right]^{T}$ is defined as the derivative of a convex function $a(z)$ with respect to the field $A(z)=\frac{\partial a}{\partial z}$. When this function equals the negative of the entropy (i.e., $a(z)=-s(z)$ ) the intensive variables are the ones included in the Gibbs-Duhem relationship Callen (1985) so that:

$$
A=\left[-\frac{1}{T},-\frac{P}{T}, \frac{\mu_{1}}{T}, \ldots, \frac{\mu_{n s}}{T}\right]^{T} .
$$

Alternatively, other criteria of interest in control, might be employed such as, for instance, the use of a quadratic function $a=z^{T} z$.

Since the function $a(z)$ is strictly convex, the map $z \longleftrightarrow A$ is one to one and onto. The same holds for deviations from arbitrary references $z^{*}, A\left(z^{*}\right)$, since by the Newton's theorem for vectorial fields (Dennis Jr. and Schnabel, 1983) we have that:

$$
A-A^{*}=Q\left(z-z^{*}\right)
$$

with

$$
Q=\int_{0}^{1} M\left(z^{*}+\varepsilon\left(z-z^{*}\right)\right) d \varepsilon ; \quad M_{i j}=\frac{\partial^{2} a(z)}{\partial z_{i} \partial z_{j}},
$$

and $\varepsilon \in[0,1]$ being a scalar parameter. Matrix $Q$, as well as $M$, is positive definite since $a(z)$ is convex (Alonso and Ydstie, 2001).

Before continuing with the description, let us introduce a new system representation that, as will be discussed in the following sections, will help handling the nonlinearity associated with the diffusion term. In this representation, the field dependent diffusion matrix $L(\cdot)$ is substituted in Eqn (1) by the corresponding Kirchhoff transformation (Saro et al., 1995; Scheerlinck et al., 2001):

$$
\Gamma=\int_{A^{0}}^{A} L(A) d A
$$

with $A^{0}$ being a given reference state. Introducing Eqn (3) into System (1) results into:

$$
\frac{\partial z}{\partial t}+\nabla(\mathbf{v} z)=\Delta \Gamma+\Sigma(z)+p
$$


The integral (3) has an unique solution provided that it is path independent. This is the case when the differential

$$
d \Gamma_{i}=\sum_{j=1}^{n s} L_{i j}(A) d A_{j}=\sum_{j=1}^{n s} \frac{\partial \Gamma_{i j}}{\partial A_{j}} d A_{j}, \quad \forall i, j=1, \ldots, n s,
$$

is exact. Such condition is satisfied whenever the following relations hold among functions $\left\{L_{i j}\right\}_{i, j=1}^{n s}$ (Smith et al., 1996):

$$
\left(\frac{\partial L_{i m}}{\partial A_{n}}\right)_{A^{[n]}}=\left(\frac{\partial L_{i n}}{\partial A_{m}}\right)_{A^{[m]}} ; \quad \forall i, m, n=1, \ldots, n s ; \quad m \neq n,
$$

where the notation $A^{[k]}$ is employed to represent the set $\left\{A_{p}\right\}_{p=1}^{n s} \backslash A_{k}$.

Note that, since $L(A)$ is positive definite and the differential is exact, the map $\Gamma \longleftrightarrow A$ is one to one and onto. In the remaining of the paper we will restrict to systems obeying condition (5), as it is characteristic of many isothermal and non-isothermal distributed reaction systems.

System (4) is defined over $\mathscr{D}=\mathscr{V} \times \mathscr{B} \cup \mathscr{T}$ where $\mathscr{V}$ is the spatial domain with smooth boundary $\mathscr{B}$ and $\mathscr{T}$ the semi-open time interval $[0, \infty)$. Functions defined on $\mathscr{V}$ are equipped with inner product and $\mathcal{L}_{2}$ norm:

$$
\langle g, h\rangle_{\mathscr{V}}=\int_{\mathscr{V}} g^{T} h d \xi, \quad\|g\|_{\mathscr{V}}=\left(\langle g, g\rangle_{\mathscr{V}}\right)^{1 / 2}
$$

For convenience, let us choose the following reference:

$$
\frac{\partial z^{*}}{\partial t}+\nabla\left(\mathbf{v} z^{*}\right)=\Delta \Gamma^{*}+\Sigma^{*}\left(z^{*}\right)+p^{*} ; \quad \Gamma^{*}=\int_{A^{0}}^{A^{*}} L(A) d A .
$$

System (4) in deviation form with respect to this reference can be rewritten as:

$$
\frac{\partial \bar{z}}{\partial t}+\nabla(\mathbf{v} \bar{z})=\Delta \bar{\Gamma}+\bar{\Sigma}\left(z, z^{*}\right)+\bar{p} .
$$

The production terms $\bar{\Sigma}(z)$ in Eqn (7) are assumed to be Lipschitz continuous. As in Alonso et al. (2004a), this is formally expressed in terms of $\bar{A}$ in the following condition:

Condition 1 There exists a positive constant $\mu$ and a reference $z^{*}$ such that

$$
\left[A-A^{*}\right]^{T}\left[\Sigma(z)-\Sigma\left(z^{*}\right)\right]+\ell_{\mu}\left(z ; z^{*}\right)=\mu\left[A-A^{*}\right]^{T}\left[A-A^{*}\right],
$$

with $\ell_{\mu}\left(z ; z^{*}\right)>0$ for every $z \neq z^{*}, \ell_{\mu}\left(z^{*} ; z^{*}\right)=0$.

The description of the system is completed with appropriate boundary conditions. To that purpose, we employ the methodology by Alonso et al. (2000) 
and split the boundary into three disjoint sets $\mathscr{B}=\mathscr{B}_{d} \cup \mathscr{B}_{0} \cup \mathscr{B}_{c}$. Second order boundary conditions are defined on $\mathscr{B}_{d}$ :

$$
\left[L(A) \frac{d \bar{A}}{d \mathbf{n}}\right]_{\mathscr{B}_{d}}=-H \bar{A},
$$

where $H$ is a positive definite matrix whose elements are the transfer coefficients. The second set $\left(\mathscr{B}_{0}\right)$ corresponds with that part associated with zero flux boundary conditions:

$$
\left[L(A) \frac{d \bar{A}}{d \mathbf{n}}\right]_{\mathscr{B}_{0}}=0 .
$$

$\mathscr{B}_{c}$ refers to the part of the boundary through which material flows with velocity $\mathbf{v}$. This boundary is divided into two disjoint sets satisfying:

$$
\begin{aligned}
& \operatorname{vn}\left(\mathscr{B}_{c}^{+}\right) \geq 0, \\
& \operatorname{vn}\left(\mathscr{B}_{c}^{-}\right) \leq 0,
\end{aligned}
$$

these conditions characterize the regions of the boundary where the material leaves and enters, respectively. Choosing the reference as the value of the field in $\mathscr{B}_{c}^{-}$, boundary conditions on this region become:

$$
\bar{z}\left(\mathscr{B}_{c}^{-}\right)=0 \text {. }
$$

\section{A Note on Dissipativity and Series Expansion of the Field}

The purpose of this section is to set up the basis for the derivation of a lowdimensional approximation of RDC systems. Since the Fourier series theorem (Reddy, 1998) plays a central role in the approach, a version of it, suitable for the approximation, will be first discussed. Next, the conditions under which the different terms of Eqn (7) can match into the theorem will be established. To that purpose, we will make use of concepts such as dissipativity and passivity as developed by Alonso and Ydstie (2001) and Alonso et al. (2004a). It must be pointed out that distributed chemical reaction systems lay into this cathegory.

Essentially, the Fourier series theorem (Reddy, 1998) establishes that given an orthonormal basis set of a Hilbert space $\mathcal{L}_{2}$ (i.e., a maximal orthonormal set $\left.\Phi=\left\{\phi_{i}\right\}_{i=1}^{\infty}\right)$ any function $f \in \mathcal{L}_{2}$ can be expanded in convergent series of the form:

$$
f(\xi, t)=\sum_{i=1}^{\infty}\left\langle\phi_{i}(\xi), f(\xi, t)\right\rangle_{\mathscr{V}} \phi_{i}(\xi)=\sum_{i=1}^{\infty} r_{i}(t) \phi_{i}(\xi) .
$$

Since the Laplacian is a particular case of the Sturm-Liouville operator, its eigenfunctions form a maximal orthonormal set for the Hilbert space $\mathcal{L}_{2}$ (Gustafson, 
1987). A possible basis set is that corresponding to the following eigenvalue problem:

$$
\Delta \phi_{i}=-\lambda_{i} \phi_{i}
$$

with appropriate boundary conditions. The eigenvalues $\left(\lambda_{i}\right)$ may be arranged so that $0 \leq \lambda_{i} \leq \lambda_{j}$ for $i<j$ and it can be shown that $\lambda_{n} \rightarrow \infty$ as $n \rightarrow \infty$ (Smoller, 1994; Reddy, 1998).

The reduced order representation of a given system is a key element in the design of our control law. The properties that allow us to obtain such representation are the unboundedness of the eigenvalues and the possibility of expanding all the terms of System (7) as in Eqn (12), so that:

$$
\begin{gathered}
\bar{z}=\sum_{i=1}^{\infty} m_{i}(t) \phi_{i}(\xi) ; \quad \bar{A}=\sum_{i=1}^{\infty} \alpha_{i}(t) \phi_{i}(\xi) ; \quad \nabla \bar{z}=\sum_{i=1}^{\infty} \tau_{i}(t) \phi_{i}(\xi) \\
\bar{\Sigma}=\sum_{i=1}^{\infty} \sigma_{i}(t) \phi_{i}(\xi) ; \quad \bar{\Gamma}=\sum_{i=1}^{\infty} \gamma_{i}(t) \phi_{i}(\xi) ; \quad \bar{p}=\sum_{i=1}^{\infty} \pi_{i}(t) \phi_{i}(\xi)
\end{gathered}
$$

The concept of passivity will be employed to show that the terms on the left hand side of relations (14) are square integrable functions (i.e., their $\mathcal{L}_{2}$ norms -as defined in Eqn (6)- are bounded). In consequence, they belong to the Hilbert space $\mathcal{L}_{2}$ and can be expanded in infinite convergent series.

First, let us state some definitions that will be employed as a part of the discussion.

Definition 1 A system is said to be passive if there exists a function $\mathcal{W}(t)$ bounded from below so that

$$
\mathcal{W}(t+T)-\mathcal{W}(t) \leq \int_{t}^{t+T}\langle y, \mathfrak{p}\rangle_{\mathscr{V}} d s, \quad \forall t, T>0
$$

with $\mathfrak{p}$ and $y$ being the input and the output of the system, respectively.

Definition 2 Consider a given convex function a(z). System (7) is said to be dissipative with respect to the function a(z) if, in Condition (1), $\ell_{0}\left(z ; z^{*}\right) \geq 0$ for $z$ such that $\left\|z-z^{*}\right\| \geq \rho$ for some positive $\rho$. Furthermore if $\ell_{0}\left(z ; z^{*}\right)>0$ for every $z \neq z^{*}$ and $\ell_{0}\left(z^{*} ; z^{*}\right)=0$ the system is purely dissipative.

Consider a system of the form (7) to be dissipative according to Definition 2 and let us split the state space into two disjoint sets:

$\Omega$ and $\mathcal{D}$, with $\Omega$ being the region where $\ell_{0} \leq 0$ and $\mathcal{D}$ its complement (i.e., the set where $\ell_{0}>0$ ). 
According to Definition 2, the norm of the field on $\Omega$ is bounded by the parameter $\rho$. Now consider the region $\mathcal{D}$. From function $a(z)$ of section 2 we can define a new convex function $w\left(z, z^{*}\right) \geq 0$. The spatial integral of $w\left(z, z^{*}\right)$ is denoted by $\mathcal{W}=\int_{\mathscr{V}} w\left(z, z^{*}\right) d \xi$ and the time derivative of $\mathcal{W}$ satisfies:

$$
\frac{d \mathcal{W}}{d t}=\langle\bar{A}, \Delta \bar{\Gamma}\rangle_{\mathscr{V}}-\langle\bar{A}, \nabla(\mathbf{v} \bar{z})\rangle_{\mathscr{V}}-L_{\mu}+\langle y, \mathfrak{p}\rangle_{\mathscr{V}}
$$

where $L_{\mu}=\int_{\mathscr{V}} \ell_{\mu} d \xi$ and $\langle\bar{A}, \Delta \bar{\Gamma}\rangle_{\mathscr{V}}-\langle\bar{A}, \nabla(\mathbf{v} \bar{z})\rangle_{\mathscr{V}} \leq 0$. The mathematical details of this derivation are part of the proof of Lemma 1 (Appendix A) collected in Appendix B. Choosing $\mu=0$, Eqn (16) becomes:

$$
\frac{d \mathcal{W}}{d t} \leq-L_{0}+\langle\bar{A}, \bar{p}\rangle_{\mathscr{V}} \Rightarrow \mathcal{W}(T) \leq \mathcal{W}(0)-\int_{0}^{T} L_{0} d t+\int_{0}^{T}\langle\bar{A}, \bar{p}\rangle_{\mathscr{V}} d t
$$

Since $\ell_{0}$ (and thus $L_{0}$ ) are positive in $\mathcal{D}$, we obtain

$$
\mathcal{W}(T) \leq \mathcal{W}(0)+\int_{0}^{T}\langle\bar{A}, \bar{p}\rangle_{\mathscr{V}} d t
$$

This implies that function $\mathcal{W}(T)$ is bounded for all $T>0$, provided that the controls are bounded so that $\int_{0}^{T}\langle\bar{A}, \bar{p}\rangle_{\mathscr{V}} d t<\infty$.

On the other hand, as it was demonstrated in Alonso and Ydstie (2001), function $w\left(z ; z^{*}\right)$ is bounded by the field as:

$$
q_{0}\left\|z-z^{*}\right\|^{2} \leq w\left(z ; z^{*}\right) \leq q_{1}\left\|z-z^{*}\right\|^{2}
$$

where $q_{0}$ and $q_{1}$ are strictly positive constants. Spatial integration of inequality (17) gives us a bound in the $\mathcal{L}_{2}$ norm: $\|\bar{z}\|_{\mathscr{V}}^{2} \leq \mathcal{W} / q_{0}<\infty$, which coincides with the conditions of the Fourier series theorem. Thus, $\bar{z}$ can be expanded as an infinite convergent series.

In order to show that the term $\nabla \bar{z}$ is also bounded, we make use of Eqn (16) and obtain

$$
\begin{gathered}
\frac{d \mathcal{W}}{d t} \leq-\langle\nabla \bar{A}, L(A) \nabla \bar{A}\rangle_{\mathscr{V}}-L_{\mu}+\langle y, \mathfrak{p}\rangle_{\mathscr{V}} \Rightarrow \\
\mathcal{W}(T)-\mathcal{W}(0) \leq-\int_{0}^{T}\langle\nabla \bar{A}, L(A) \nabla \bar{A}\rangle_{\mathscr{V}} d s+\int_{0}^{T}\left(-L_{\mu}+\langle y, \mathfrak{p}\rangle_{\mathscr{V}}\right) d s .
\end{gathered}
$$

$L(A)$ is positive definite and therefore there exists a constant $\delta_{1}>0$ such that $\delta_{1}\|\nabla \bar{A}\|_{\mathscr{V}}^{2} \leq\langle\nabla \bar{A}, L(A) \nabla \bar{A}\rangle_{\mathscr{V}}$. With this inequality Eqn (18) becomes

$$
\delta_{1}\|\nabla \bar{A}\|_{\mathscr{V}}^{2} \leq \mathcal{W}(0)-\mathcal{W}(T)+\int_{0}^{T}\left(-L_{\mu}+\langle y, \mathfrak{p}\rangle_{\mathscr{V}}\right) d s
$$

Since the RHS is bounded for $\mu=0$ we conclude that $\|\nabla \bar{A}\|_{\mathscr{V}}^{2}<\infty$.

So far we have shown that the field and its gradient is bounded. Now, this fact will be employed next to extend the proof to the rest of terms of system (7), mainly $\bar{\Sigma}, \bar{\Gamma}$ and $\bar{p}$. 
As stated in Condition 1, the production terms $(\bar{\Sigma})$ are Lipschitz so they are bounded by the field and thus they belong to $\mathcal{L}_{2}$. On the other hand, we concentrate on systems which satisfy

$$
\langle\bar{\Gamma}, \bar{\Gamma}\rangle_{\mathscr{V}}=\|\bar{\Gamma}\|_{\mathscr{V}}^{2} \leq k_{1}^{2}\|\bar{A}\|_{\mathscr{V}}^{2}
$$

where $k_{1}$ is a given positive parameter. In this way, function $\bar{\Gamma}$ can also be expanded in infinite series of the form (14).

Note that the conditions just described are satisfied by most distributed chemical reaction systems, and thus allowing expansions of the form (14).

Finally, we restrict the class of control inputs to that bounded in the $\mathcal{L}_{2}$ norm $\left(\|\bar{p}\|_{\mathscr{V}}^{2}<\infty\right)$. This way control actions will satisfy conditions of the Fourier series theorem and will accept a convergent series expansion.

We end up this section with an example which illustrates Kirchoff transform and the notion of dissipation.

Example 1 Reaction-Diffusion system with Variable Diffusivity:

This example is a modified version of the case study described in Antoniades and Christofides (2000). The system consists of a RD process where the exothermic zeroth order reaction $A \rightarrow B$ takes place. The spatial domain is defined as $\mathscr{V}=\{\xi / 0<\xi<L\}$ with boundary $\mathscr{B}=\{0, L\}$. For this example, the temperature distribution, expressed in terms of a field $\bar{u}$, satisfies:

$$
\frac{\partial \bar{u}}{\partial t}=\nabla[D \nabla \bar{u}]+\Sigma+\beta_{H} p ; \quad \Sigma=\beta_{U}\left[\exp \left(-\frac{\gamma}{1+\bar{u}}\right)-\exp (-\gamma)\right]-\beta_{H} \bar{u},
$$

where $D=1-\alpha \bar{u}$ represents the field dependent diffusion coefficient. $\gamma$ indicates the activation energy, and $\beta_{T}, \beta_{H}$ denote the dimensionless heat of reaction and heat transfer coefficient, respectively. Finally, $p$ is the control variable. System description is completed with boundary and initial conditions of the form:

$$
\left.\bar{u}\right|_{\mathscr{B}}=0 ; \quad \bar{u}(\xi, 0)=\bar{u}_{0} .
$$

The values of the parameters and initial conditions are

$$
L=\pi, \alpha=0.05, \beta_{U}=2, \beta_{H}=50, \gamma=4, \bar{u}_{0}=0.4 \cdot \sin (2 \xi) .
$$

The field $(\bar{u})$ has a lower bound of $\bar{u}=-1$ (Alonso et al., 2002c). In addition, the upper bound of $\bar{u}$ must be 20 in order to the diffusivity to be positive.

The Kirchhoff transform (3) applied to this system results into:

$$
\Gamma=\int_{\bar{u}^{0}=0}^{\bar{u}} D d \bar{u}=\int_{\bar{u}^{0}=0}^{\bar{u}}(1-\alpha \bar{u}) d \bar{u}=\bar{u}-\frac{\alpha}{2} \bar{u}^{2},
$$


note that since $\nabla \Gamma=D \nabla \bar{u}$, then $\Delta \Gamma=\nabla(D \nabla \bar{u})$ and Eqn. (19) becomes

$$
\frac{\partial \bar{u}}{\partial t}=\Delta \Gamma+\Sigma+\beta_{H} p
$$

The results obtained by solving PDEs (19) and (21) for $p=0$ are depicted in Figures 1(a) and 1(b), respectively, showing that both representations are equivalent.

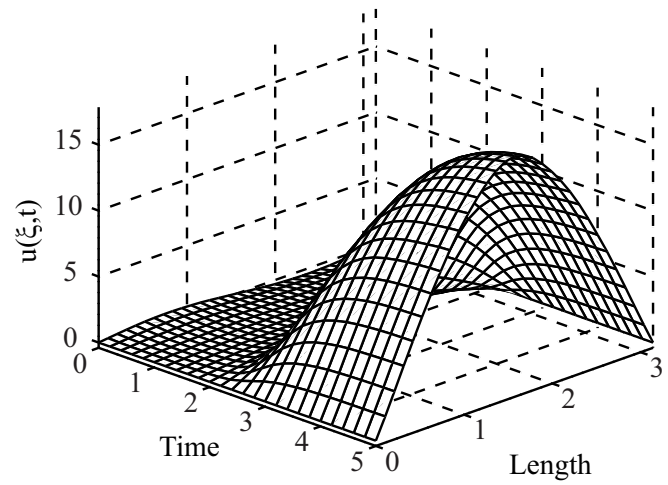

(a)

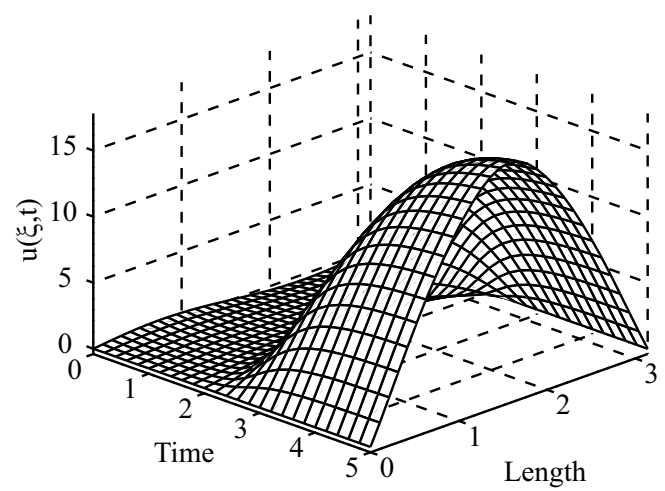

(b)

Fig. 1. Evolution of the distributed temperature. (a) Computed from system (19), (b) computed using the Kirchhoff transform -system (21)-.

To show that this system is dissipative according to Definition 2, we choose the convex function $a(\bar{u})=\frac{1}{2} \bar{u}^{2}$. The dual of the field is computed from $a(\bar{u})$ as $\bar{A}=\frac{\partial a}{\partial \bar{u}}=\bar{u}$. Finally, from Condition 1, we obtain an expression for the limit set of the form $\ell_{0}=-\bar{A} \Sigma=-\bar{u} \Sigma$. Figure 2 depicts the value of $\ell_{0}$ as a function of $\bar{u}$. The picture shows that $\ell_{0}$ is negative only in a bounded interval

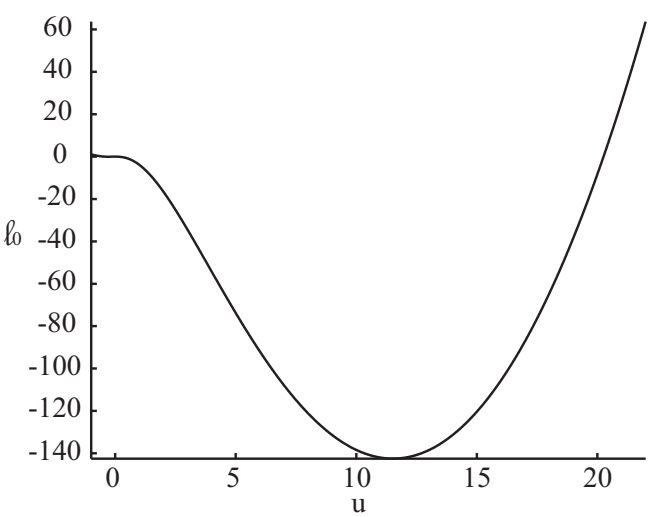

Fig. 2. Limit set $\ell_{0}$ for system (21).

of the state space so the system is dissipative with respect to $a=\frac{1}{2} \bar{u}^{2}$ but not purely dissipative. It must be pointed out that other convex functions could be employed Alonso et al. (2002c) 


\section{Approximation of RDC systems by a finite dimensional set of ODEs}

In this section, and using the concepts discussed previously, we will show that the series expansions in (14) can be separated in two summations: one containing an infinite number of elements, which will be exponentially stable, and the other containing the finite remaining ones, which will capture the relevant dynamics of the process and constitute the basis for the low dimensional approximation.

Let $S=\left\{\phi_{i}\right\}_{i=1}^{\infty}$ and $\Lambda=\left\{\lambda_{i}\right\}_{i=1}^{\infty}$ be, respectively, the complete sets of eigenfunctions and their associated eigenvalues satisfying (13). Following the methodology employed in Vilas et al. (2006), we define the subsets of natural numbers $\mathcal{N}_{a}$ and $\mathcal{N}_{b}$, with $\mathcal{N}_{a}$ being a finite subset of arbitrary numbers and $\mathcal{N}_{b}=\mathbb{N} \backslash \mathcal{N}_{a}$ its complement. These sub-sets will allow us to partition the eigenset $(S, \Lambda, \mathbb{N})$ in two disjoint sets $\left(S_{a}, \Lambda_{a}, \mathcal{N}_{a}\right)$ and $\left(S_{b}, \Lambda_{b}, \mathcal{N}_{b}\right)$ with $S_{a}=\left\{\phi_{i}\right\}_{i \in \mathcal{N}_{a}}, \Lambda_{a}=\left\{\lambda_{i}\right\}_{i \in \mathcal{N}_{a}}$ and $S_{b}$ and $\Lambda_{b}$ their corresponding complements. Using these subsets, all the terms of Eqn (4) can be split as:

$$
\begin{gathered}
\bar{z}=\bar{z}_{a}+\bar{z}_{b}=\sum_{i \in \mathcal{N}_{a}} m_{i}(t) \phi_{i}(\xi)+\sum_{i \in \mathcal{N}_{b}} m_{i}(t) \phi_{i}(\xi), \\
\bar{A}=\bar{A}_{a}+\bar{A}_{b}=\sum_{i \in \mathcal{N}_{a}} \alpha_{i}(t) \phi_{i}(\xi)+\sum_{i \in \mathcal{N}_{b}} \alpha_{i}(t) \phi_{i}(\xi), \\
\nabla \bar{z}=\nabla \bar{z}_{a}+\nabla \bar{z}_{b}=\sum_{i \in \mathcal{N}_{a}} \tau_{i}(t) \phi_{i}(\xi)+\sum_{i \in \mathcal{N}_{b}} \tau_{i}(t) \phi_{i}(\xi), \\
\bar{\Sigma}=\bar{\Sigma}_{a}+\bar{\Sigma}_{b}=\sum_{i \in \mathcal{N}_{a}} \sigma_{i}(t) \phi_{i}(\xi)+\sum_{i \in \mathcal{N}_{b}} \sigma_{i}(t) \phi_{i}(\xi), \\
\bar{\Gamma}=\bar{\Gamma}_{a}+\bar{\Gamma}_{b}=\sum_{i \in \mathcal{N}_{a}} \gamma_{i}(t) \phi_{i}(\xi)+\sum_{i \in \mathcal{N}_{b}} \gamma_{i}(t) \phi_{i}(\xi), \\
\bar{p}=\bar{p}_{a}+\bar{p}_{b}=\sum_{i \in \mathcal{N}_{a}} \pi_{i}(t) \phi_{i}(\xi)+\sum_{i \in \mathcal{N}_{b}} \pi_{i}(t) \phi_{i}(\xi),
\end{gathered}
$$

The following Proposition establishes the conditions for constructing low dimensional approximations of dissipative systems. The proof of this proposition can be found in Appendix E.

Proposition 1 Consider a particular subset $\left(S_{a}, \Lambda_{a}, \mathcal{N}_{a}\right)$ as that containing the $n$ smallest eigenvalues and their associated eigenfunctions. Then, if $n$ is large enough, the subfield $\left(\bar{z}_{b}\right)$ associated with the subset $\left(S_{b}, \Lambda_{b}, \mathcal{N}_{b}\right)$ is exponentially stable. In addition, the larger the value of $n$, the faster the exponential decaying of the dynamic modes of the stable subsystem.

As a consequence of the fast decaying of the modes corresponding to the set $\left(S_{b}, \Lambda_{b}, \mathcal{N}_{b}\right)$, the series expansion (22) can be truncated to obtain an approxi- 
mated solution of the form:

$$
\bar{z}(\xi, t) \approx \widetilde{z}(\xi, t)=\sum_{i \in \mathcal{N}_{a}} m_{i}(t) \phi_{i}(\xi)
$$

which constitutes the basis for the reduced order model (ROM) development (Shvartsman and Kevrekidis, 1998; Alonso et al., 2004b). Usually the number of modes necessary to obtain a good approximation is much lower than the number of equations required in classical methods such as finite elements or finite differences. This property will be employed to derive the control laws in Section 5.

The ROM is obtained by projecting PDE (7) over the eigenfunctions of the set $S_{a}$ so that:

$$
\left\langle\Phi_{a}, \frac{\partial \bar{z}}{\partial t}\right\rangle_{\mathscr{V}}+\left\langle\Phi_{a}, \nabla(\mathbf{v} \bar{z})\right\rangle_{\mathscr{V}}=\left\langle\Phi_{a}, \Delta \bar{\Gamma}\right\rangle_{\mathscr{V}}+\left\langle\Phi_{a}, \bar{\Sigma}\right\rangle_{\mathscr{V}}+\left\langle\Phi_{a}, \bar{p}\right\rangle_{\mathscr{V}}
$$

with $\Phi_{a}=\left[\phi_{\mathcal{N}_{a}(1)}, \ldots, \phi_{\mathcal{N}_{a}(n)}\right]$. Applying expressions (22)-(27) to Eqn (29), results into:

$$
\begin{gathered}
\left\langle\Phi_{a}, \sum_{i \in \mathcal{N}_{a}} \phi_{i} \frac{d m_{i}}{d t}+\sum_{i \in \mathcal{N}_{b}} \phi_{i} \frac{d m_{i}}{d t}\right\rangle_{\mathscr{V}}+\left\langle\Phi_{a}, \sum_{i \in \mathcal{N}_{a}} \phi_{i} \tau_{i}+\sum_{i \in \mathcal{N}_{b}} \phi_{i} \tau_{i}\right\rangle_{\mathscr{V}}= \\
-\left\langle\Phi_{a}, \sum_{i \in \mathcal{N}_{a}} \phi_{i} \lambda_{i} \gamma_{i}+\sum_{i \in \mathcal{N}_{b}} \phi_{i} \lambda_{i} \gamma_{i}\right\rangle_{\mathscr{V}}+\left\langle\Phi_{a}, \sum_{i \in \mathcal{N}_{a}} \phi_{i} \sigma_{i}+\sum_{i \in \mathcal{N}_{b}} \phi_{i} \sigma_{i}\right\rangle_{\mathscr{V}}+ \\
\left\langle\Phi_{a}, \sum_{i \in \mathcal{N}_{a}} \phi_{i} \pi_{i}+\sum_{i \in \mathcal{N}_{b}} \phi_{i} \pi_{i}\right\rangle_{\mathscr{V}} .
\end{gathered}
$$

Finally, and since the eigenfunctions are orthonormal, the previous expression becomes:

$$
\frac{d m_{a}}{d t}=-\Upsilon_{a} \gamma_{a}-\tau_{a}+\sigma_{a}+\pi_{a}
$$

where $\Upsilon_{a}$ is a diagonal matrix containing the eigenvalues of the set $\Lambda_{a}$.

The global spatial dependent functions in Eqn (28) were obtained by solving the eigenvalue problem (13). It must be pointed out that alternative functions could be employed in the approximation. In this context, the Proper Orthogonal Decomposition (POD) method (Sirovich, 1987; Holmes et al., 1997) provides a set of empirical basis functions which are optimal with respect to other possible expansions. This set is optimal in the sense that, for a given number of basis functions, it captures most of the relevant dynamic behavior of the original distributed system in the range of initial conditions, parameters, inputs, and/or perturbations of the experimental data (Balsa-Canto et al., 2004). In this work, the set of eigenfunctions computed from the eigenvalue problem (13) was chosen among other alternatives (e.g. the POD method) because this particular basis set allows us to establish a straightforward relationship between rate of convergence and amount of dissipation (Alonso et al., 2004a). 


\section{Robust Stabilization of Reaction-Diffusion-Convection Systems}

The aim of this section is to design a class of controllers able to force RDC systems to follow a given reference trajectory (the tracking control problem). In addition, the control law will be constructed so as to stabilize the reference without the need of detailed information on the structure of nonlinear terms and/or in the system parameters (robustness). To that purpose, we will combine classical results on the robust nonlinear control of finite dimensional systems, in particular the Lyapunov redesign technique (Khalil, 1996), with the low dimensional approximations described in Section 4. The effect of this approach is sketched in Figure 3. Here, the state is represented in deviation

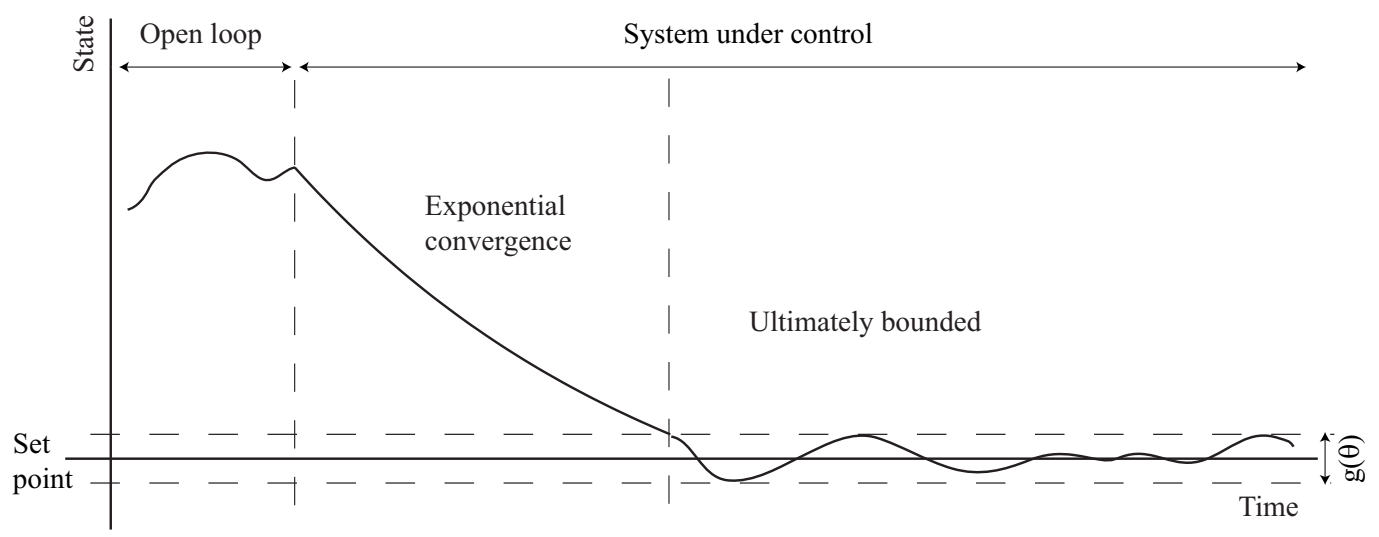

Fig. 3. Evolution of a given system with uncertainties under a control law constructed using the Lyapunov redesign technique.

form with respect to a reference (error). When the control law enters in action (first vertical dashed line), it drives the error exponentially fast to a region close to the reference. Once the error reaches this region, it will remain there in the future (ultimately bounded). Note that, since uncertainties are considered, the convergence to the set point cannot be ensured but only convergence to an arbitrarily small region around it.

Consider system (4) and the reference

$$
\frac{\partial z^{*}}{\partial t}=\Delta \Gamma^{*}-\nabla\left(\mathbf{v} z^{*}\right)+\Sigma^{*}+p^{*}, \quad \Gamma^{*}=\int_{A^{0}}^{A^{*}} L^{*}(A) d A .
$$

Note that this can be a non stationary reference and the term $L^{*}(A)$ can be different to $L(A)$. The system in deviation form is rewritten as

$$
\frac{\partial \bar{z}}{\partial t}=\Delta \Gamma-\Delta \Gamma^{*}-\nabla(\mathbf{v} \bar{z})+\bar{\Sigma}+\bar{p}
$$

Let us operate the term $\Gamma-\Gamma^{*}$ :

$$
\Gamma-\Gamma^{*}=\int_{A^{0}}^{A} L(A) d A-\int_{A^{0}}^{A^{*}} L^{*}(A) d A=\int_{A^{0}}^{A} L(A) d A-\int_{A^{0}}^{A^{*}} L^{*}(A) d A+
$$




$$
\begin{gathered}
\int_{A^{0}}^{A^{*}} L(A) d A-\int_{A^{0}}^{A^{*}} L(A) d A=\int_{A^{*}}^{A} L(A) d A-\int_{A^{0}}^{A^{*}} L^{*}(A)-L(A) d A= \\
\int_{A^{*}}^{A} L(A) d A-\int_{A^{0}}^{A^{*}} \chi(A) d A=\bar{\Gamma}-\Gamma_{d}
\end{gathered}
$$

where $\chi(A)=L^{*}(A)-L(A)$. Substituting this expression into equation (30):

$$
\frac{\partial \bar{z}}{\partial t}=\Delta \bar{\Gamma}-\Delta \Gamma_{d}-\nabla(\mathbf{v} \bar{z})+\bar{\Sigma}+\bar{p}
$$

Making use of previous results, the set of modes for the reference trajectory $m^{*}$ will be separated as follows:

$$
m^{*}=\left[\begin{array}{l}
m_{a}^{*} \\
m_{b}^{*}
\end{array}\right]
$$

The set $m_{b}^{*}=\left[m_{\mathcal{N}_{b}(1)}^{*}, m_{\mathcal{N}_{b}(2)}^{*}, \ldots\right]^{T}$ is composed of the exponentially stable modes (non representative modes) while the finite remaining ones (representative modes) are included into the set $m_{a}^{*}$. The same separation procedure will be applied to the modes of the system to be controlled $(m)$. The control law will be then designed so as to achieve two different objectives: on the one hand the stabilization of modes $\left(m_{b}, \mathcal{N}_{b}\right)$ and on the other hand, to force the remaining $\left(m_{a}, \mathcal{N}_{a}\right)$ to follow the reference trajectory $\left(m_{a}^{*}, \mathcal{N}_{a}\right)$.

In order to construct the control logic, first Eqn. (31) is projected over the eigenfunctions $\Phi_{a}$ and $\Phi_{b}$, this leading to

$$
\begin{aligned}
\left\langle\Phi_{a}, \frac{\partial \bar{z}}{\partial t}\right\rangle_{\mathscr{V}}=\frac{d \bar{m}_{a}}{d t}= & \left\langle\Phi_{a}, \Delta \bar{\Gamma}\right\rangle_{\mathscr{V}}-\left\langle\Phi_{a}, \Delta \Gamma_{d}\right\rangle_{\mathscr{V}}-\left\langle\Phi_{a}, \nabla(\mathbf{v} \bar{z})\right\rangle_{\mathscr{V}}+ \\
& \left\langle\Phi_{a}, \bar{\Sigma}\right\rangle_{\mathscr{V}}+\left\langle\Phi_{a}, \bar{p}\right\rangle_{\mathscr{V}} \\
\left\langle\Phi_{b}, \frac{\partial \bar{z}}{\partial t}\right\rangle_{\mathscr{V}}=\frac{d \bar{m}_{b}}{d t}= & \left\langle\Phi_{b}, \Delta \bar{\Gamma}\right\rangle_{\mathscr{V}}-\left\langle\Phi_{b}, \Delta \Gamma_{d}\right\rangle_{\mathscr{V}}-\left\langle\Phi_{b}, \nabla(\mathbf{v} \bar{z})\right\rangle_{\mathscr{V}}+ \\
& \left\langle\Phi_{b}, \bar{\Sigma}\right\rangle_{\mathscr{V}}+\left\langle\Phi_{b}, \bar{p}\right\rangle_{\mathscr{V}}
\end{aligned}
$$

Since the modes $m_{b}^{*}$ are exponentially stable they can be neglected, this implying that $\Delta \Gamma_{b}^{*} \approx 0$, and the previous expression can be rewritten as:

$$
\frac{d \bar{m}_{b}}{d t}=\left\langle\Phi_{b}, \Delta \Gamma\right\rangle_{\mathscr{V}}-\left\langle\Phi_{b}, \nabla(\mathbf{v} \bar{z})\right\rangle_{\mathscr{V}}+\left\langle\Phi_{b}, \bar{\Sigma}\right\rangle_{\mathscr{V}}+\left\langle\Phi_{b}, \bar{p}\right\rangle_{\mathscr{V}}
$$

Given that $\bar{m}_{a}=m_{a}-m_{a}^{*}$ and $\bar{m}_{b}=m_{b}-m_{b}^{*}$, a control law which stabilizes $\bar{m}_{a}$ and $\bar{m}_{b}$ will make $m_{a} \rightarrow m_{a}^{*}, m_{b} \rightarrow m_{b}^{*}=0$ and thus $z \rightarrow z^{*}$.

The next step is to construct adequate Lyapunov functions $\mathcal{W}_{a}$ and $\mathcal{W}_{b}$ and to obtain an expression for their time derivatives. We concentrate now on $\mathcal{W}_{a}$ since the same procedure can be applied so as to obtain $\mathcal{W}_{b}$. Multiplying Eqn 
(32) by the time dependent functions of Eqn (23) $-\alpha_{a}^{T}$ :

$$
\alpha_{a}^{T} \frac{d \bar{m}_{a}}{d t}=\left\langle\bar{A}_{a}, \Delta \bar{\Gamma}\right\rangle_{\mathscr{V}}-\left\langle\bar{A}_{a}, \Delta \Gamma_{d}\right\rangle_{\mathscr{V}}-\left\langle\bar{A}_{a}, \nabla(\mathbf{v} \bar{z})\right\rangle_{\mathscr{V}}+\left\langle\bar{A}_{a}, \bar{\Sigma}\right\rangle_{\mathscr{V}}+\left\langle\bar{A}_{a}, \bar{p}\right\rangle_{\mathscr{V}}
$$

Substituting expressions (5)-(8) of Lemma 2 -see Appendix C- into equation (34), it becomes:

$$
\alpha_{a}^{T} \frac{d \bar{m}_{a}}{d t} \leq-\zeta \lambda_{m} \alpha_{a}^{T} \alpha_{a}+\lambda_{q}\left\|\gamma_{d a}\right\|_{2}\left\|\bar{A}_{a}\right\|_{\mathscr{V}}+\eta_{a}\left\|\bar{A}_{a}\right\|_{\mathscr{V}}+\left\langle\bar{A}_{a}, \bar{p}_{a}\right\rangle_{\mathscr{V}}
$$

A new convex function $w_{a}$ is defined as in Eqn. (B.1) -see Appendix B- for the field $\bar{z}_{a}$. The time derivative of $w_{a}$ now reads:

$$
\frac{\partial w_{a}}{\partial t}=\frac{\partial w_{a}}{\partial \bar{z}_{a}} \frac{\partial \bar{z}_{a}}{\partial t}=\bar{A}_{a}^{T} \frac{\partial \bar{z}_{a}}{\partial t} \Rightarrow \frac{d \mathcal{W}_{a}}{d t}=\int_{\mathscr{V}} \frac{\partial w_{a}}{\partial t} d \xi=\alpha_{a}^{T} \frac{d \bar{m}_{a}}{d t} .
$$

In addition, $\bar{A}_{a}$ relates to $\bar{z}_{a}$ through an expression of the form (2) so that

$$
\alpha_{a}^{T} \alpha_{a}=\left\langle\bar{A}_{a}, \bar{A}_{a}\right\rangle_{\mathscr{V}}=\left\langle Q \bar{z}_{a}, Q \bar{z}_{a}\right\rangle_{\mathscr{V}} \geq \delta_{0}^{2} m_{a}^{T} m_{a} \geq \frac{\delta_{0}^{2}}{q_{1}} \mathcal{W}_{a}
$$

where $\delta_{0}$ is the minimum eigenvalue of $Q$. Using these relations, inequality (35) can be rewritten as:

$$
\frac{d \mathcal{W}_{a}}{d t} \leq-\zeta \lambda_{m} \frac{\delta_{0}^{2}}{q_{1}} \mathcal{W}_{a}+\lambda_{q}\left\|\gamma_{d a}\right\|_{2}\left\|\bar{A}_{a}\right\|_{\mathscr{V}}+\eta_{a}\left\|\bar{A}_{a}\right\|_{\mathscr{V}}+\left\langle\bar{A}_{a}, \bar{p}_{a}\right\rangle_{\mathscr{V}}
$$

The last step consists of selecting a control law in order to cancel the second and the third terms of the RHS of Eqn (36). The following proposition suggests a possible controller that attains such objective.

Proposition 2 A control law of the form

$$
\bar{p}_{a}=\left\{\begin{array}{lcc}
-\omega_{a} \frac{\bar{A}_{a}}{\left\|\bar{A}_{a}\right\|_{\mathscr{V}}}-\omega_{a}^{*} \frac{\bar{A}_{a}}{\left\|\bar{A}_{a}\right\|_{\mathscr{V}}} \quad \text { if } & \omega_{a}\left\|\bar{A}_{a}\right\|_{\mathscr{V}} \geq \theta_{a}, \omega_{a}^{*}\left\|\bar{A}_{a}\right\|_{\mathscr{V}} \geq \theta_{a} \\
-\omega_{a} \frac{\bar{A}_{a}}{\left\|\bar{A}_{a}\right\|_{\mathscr{V}}}-\left(\omega_{a}^{*}\right)^{2} \frac{\bar{A}_{a}}{\theta_{a}} & \text { if } & \omega_{a}\left\|\bar{A}_{a}\right\|_{\mathscr{V}} \geq \theta_{a}, \omega_{a}^{*}\left\|\bar{A}_{a}\right\|_{\mathscr{V}}<\theta_{a} \\
-\left(\omega_{a}\right)^{2} \frac{\bar{A}_{a}}{\theta_{a}}-\omega_{a}^{*} \frac{\bar{A}_{a}}{\left\|\bar{A}_{a}\right\|_{\mathscr{V}}} & \text { if } & \omega_{a}\left\|\bar{A}_{a}\right\|_{\mathscr{V}}<\theta_{a}, \omega_{a}^{*}\left\|\bar{A}_{a}\right\|_{\mathscr{V}} \geq \theta_{a} \\
-\left(\omega_{a}\right)^{2} \frac{\bar{A}_{a}}{\theta_{a}}-\left(\omega_{a}^{*}\right)^{2} \frac{\bar{A}_{a}}{\theta_{a}} & \text { if } & \omega_{a}\left\|\bar{A}_{a}\right\|_{\mathscr{V}}<\theta_{a}, \omega_{a}^{*}\left\|\bar{A}_{a}\right\|_{\mathscr{V}}<\theta_{a}
\end{array}\right.
$$

where $\omega_{a}$ and $\omega_{a}^{*}$ are known functions which satisfy $\omega_{a} \geq \lambda_{q}\left\|\gamma_{d a}\right\|_{2}$ and $\omega_{a}^{*} \geq \eta_{a}$, will make the field $\bar{z}_{a}$ to be ultimately bounded. 
The proof of this Proposition can be found in Appendix F.

As mentioned above, the same procedure can be applied to obtain an expression of the form of (36) for the Lyapunov function $\mathcal{W}_{b}$ :

$$
\frac{d \mathcal{W}_{b}}{d t} \leq-\zeta \lambda_{m} \frac{\delta_{0}^{2}}{q_{1}} \mathcal{W}_{b}+\eta_{b}\left\|\bar{A}_{b}\right\|_{\mathscr{V}}+\left\langle\bar{A}_{b}, \bar{p}_{b}\right\rangle_{\mathscr{V}}
$$

where $\eta_{b}>\left\|\bar{\Sigma}_{b}\right\|_{\mathscr{V}}$

Proposition 3 A control law of the form:

$$
\bar{p}_{b}=\left\{\begin{array}{lll}
-\omega_{b}^{*} \frac{\bar{A}_{b}}{\left\|\bar{A}_{b}\right\|_{\mathscr{V}}} & \text { if } & \omega_{b}^{*}\left\|\bar{A}_{b}\right\|_{\mathscr{V}} \geq \theta_{b} \\
-\left(\omega_{b}^{*}\right)^{2} \frac{\bar{A}_{b}}{\theta_{a}} & \text { if } & \omega_{b}^{*}\left\|\bar{A}_{b}\right\|_{\mathscr{V}}<\theta_{b}
\end{array}\right.
$$

with $\omega_{b}^{*} \geq \eta_{b}$, will make the fiel $\bar{z}_{b}$ to be ultimately bounded.

The same line of arguments employed in the proof of Proposition 2 can be applied in a straightforward manner to show that the control law (39) stabilizes $\left(m_{b}, \mathcal{N}_{b}\right)$. The proof of Proposition 2 also shows that the price to pay for robustness is that asymptotic convergence cannot be ensured but only convergence to a region around the reference. It must be pointed out that this region can be arbitrarily reduced by decreasing the control parameters $\theta_{a}$ and $\theta_{b}$ but at the cost of larger control efforts. This fact will be illustrated in the next section.

\section{Computational Experiments}

In order to illustrate the main aspects of the results presented so far, we consider two computational experiments. In the first one, a tubular reactor with variable diffusivity is employed to show the importance of choosing an adequate convex function $w$ for control purposes. In the second case, the objective is to design a control law able to stabilize a given complex behavior (limit cycle) in RDC systems with uncertainties.

\subsection{Tubular reactor with field dependent diffusivity}

The system considered here is that described in Example 1 of Section 3. In this process, the heat produced by the reaction is removed from the system through 
a cooling jacket. The interaction between the diffusion, the reaction heat and the cooling medium can produce a runaway phenomena. The objective is to construct a control law able to stabilize the system.

For convenience, let us rewrite the model equations:

$$
\frac{\partial \bar{u}}{\partial t}=\Delta \Gamma+\Sigma+\beta_{H} p ; \quad \Sigma=\beta_{U}\left[\exp \left(-\frac{\gamma}{1+\bar{u}}\right)-\exp (-\gamma)\right]-\beta_{H} \bar{u}
$$

with boundary and initial conditions

$$
\left.\bar{u}\right|_{\mathscr{B}}=0 ; \quad \bar{u}(\xi, 0)=\bar{u}_{0} .
$$

The controller is now designed as follows: first, we choose a convex function $w$ and then calculate its time derivative along the trajectories (40) so that:

$$
\frac{d w}{d t}=\bar{A}^{T} \frac{\partial \bar{u}}{\partial t}=\bar{A}^{T} \Delta \Gamma+\bar{A}^{T} \Sigma+\beta_{H} \bar{A}^{T} p
$$

where $\bar{A}=\frac{\partial w}{\partial \bar{u}}$. Integrating over the spatial domain and denoting $\mathcal{W}=\int_{\mathscr{V}} w d \xi$ :

$$
\frac{d \mathcal{W}}{d t}=\langle\bar{A}, \Delta \Gamma\rangle_{\mathscr{V}}+\langle\bar{A}, \Sigma\rangle_{\mathscr{V}}+\beta_{H}\langle\bar{A}, p\rangle_{\mathscr{V}}
$$

As shown in Section 3, the first term of the RHS is non positive. Combining this with Eqn (8), we can rewrite Eqn (42) as:

$$
\frac{d \mathcal{W}}{d t} \leq \mu\langle\bar{A}, \bar{A}\rangle_{\mathscr{V}}-L_{\mu}+\beta_{H}\langle\bar{A}, p\rangle_{\mathscr{V}}
$$

Condition 1 establishes that there exists a $\mu>0$ for which $L_{\mu}>0$ so that

$$
\frac{d \mathcal{W}}{d t} \leq \mu\langle\bar{A}, \bar{A}\rangle_{\mathscr{V}}+\beta_{H}\langle\bar{A}, p\rangle_{\mathscr{V}}
$$

Choosing a control law of the form $p=-\frac{\omega}{\beta_{H}} \bar{A}$ with $\omega>\mu$ and using expressions (2) and (17) it is found that $\frac{d \mathcal{W}}{d t} \leq \chi \mathcal{W}$ where $\chi=\frac{\delta_{0}^{2}}{q_{1}}(\mu-\omega)<0$ and using the Gronwall-Bellman lemma (Khalil, 1996) we have that:

$$
\mathcal{W}(t) \leq \mathcal{W}(0) \exp (\chi t)
$$

which implies that the field $\bar{u}$ evolves exponentially to zero.

The dual of the field $(\bar{A})$ is closely connected with the convex function $w$ so the control effort will strongly depend on the selection of such a function. In order to illustrate this point, we have considered two possibilities:

$$
w_{1}=\frac{1}{2} \bar{u}^{2}
$$


and

$$
w_{2}=\frac{1}{2} \bar{u}^{2}-1-\sigma \bar{u}+\exp (\sigma \bar{u})
$$

where $\sigma$ is a positive design parameter. $w_{2}$ was selected so as to take into account the structure of the nonlinear term as proposed in Alonso et al. (2002a). The intensive counterparts of the field for $w_{1}$ and $w_{2}$ are:

$$
\begin{gathered}
\bar{A}_{1}=\bar{u} \\
\bar{A}_{2}=\bar{u}+\sigma[-1+\exp (\sigma \bar{u})] .
\end{gathered}
$$

Using Eqn (8) one can check that for $\mu_{1}>4.155$ and $\mu_{2}>0.99$ with $\sigma=1.26$, $\ell_{\mu}$ is positive for all $\bar{u}$. The gains $\omega$ in the control law are chosen according to these values of $\mu$.

Figures 4(a) and 4(b) show the evolution of the field under the control $p_{i}=$ $-\frac{\omega}{\beta_{H}} \bar{A}_{i}$ with $i=1$ and $i=2$, respectively. The effort of both control laws is represented in Figure 4(c). As shown in the Figure, using the function $w_{2}$ (dashed line) results into smoother control actions as compared with $w_{1}$ (continuous line). This indicates that, although any convex positive definite function $w$ could be employed, it may be worth searching for a given $w$ capturing the nonlinearity of the process, especially when considering bounds on the controls.

\subsection{Nonlinear Reaction-Convection-Diffusion Process}

This case study corresponds with a non-isothermal tubular reactor where the exothermic first order reaction $A \rightarrow B$ takes place. The heat produced by the reaction is removed from the medium through a cooling jacket and a portion of the product stream is recycled to the fresh feed. The dynamics of the system is described by the following set of coupled nonlinear PDEs (Antoniades and Christofides, 2001):

$$
\frac{\partial z(t, \xi)}{\partial t}=D \Delta z(t, \xi)-\nabla z(t, \xi)+\Sigma(z)+p(t, \xi)
$$

defined on the $1 \mathrm{D}$ spatial domain $\mathscr{V}=\{\xi / 0<\xi<1\}$ with boundary $\mathscr{B}=\{0,1\} \cdot z=[C, T]^{T}$ is the vector field where $C$ and $T$ represent the concentration of the specie $A$ and temperature of the medium, respectively, in deviation form with respect to a stationary state. $D$ is a diagonal matrix whose elements are the diffusion coefficients:

$$
D=\left[\begin{array}{cc}
\frac{1}{P e_{C}} & 0 \\
0 & \frac{1}{P e_{T}}
\end{array}\right] .
$$




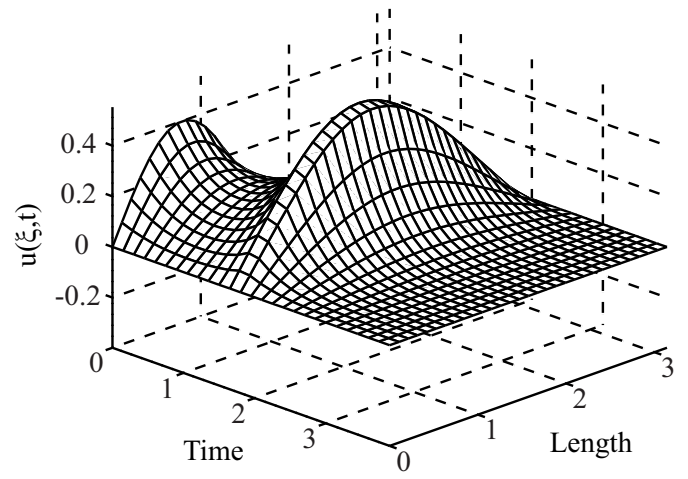

(a)

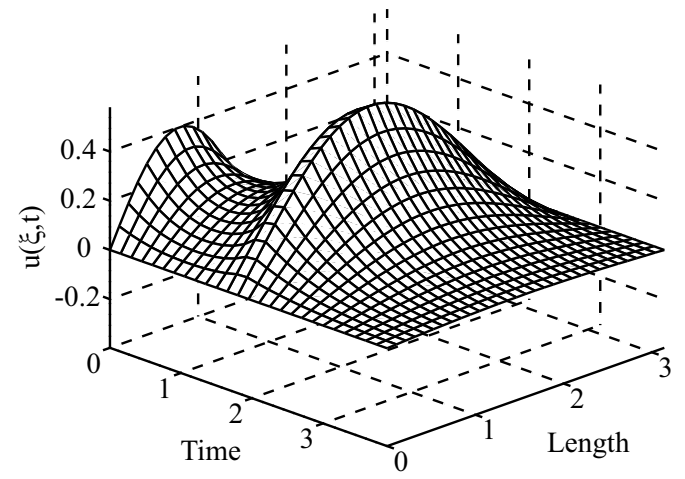

(b)

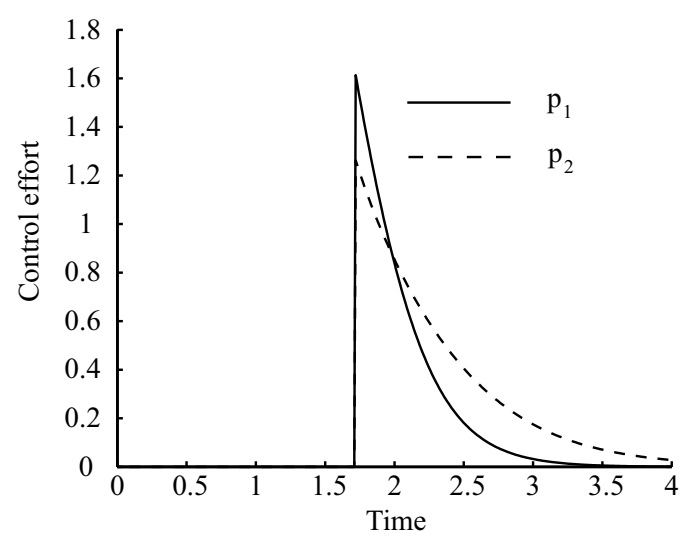

(c)

Fig. 4. Evolution of the field under a control law designed by using different convex functions (a) using function $w_{1}$, (b) using function $w_{2}$. (c) Control effort for both functions.

$\Sigma(z)$ denotes the reaction term of the form:

$$
\Sigma(z)=\left[\begin{array}{c}
-f(C, T) \\
B_{T} f(C, T)-\beta_{T} T
\end{array}\right] ; \quad f(C, T)=B_{C}(1+C) \exp \left(\frac{\gamma T}{1+T}\right) .
$$

Finally, $p$ stands for the control term:

$$
p=\left[\begin{array}{c}
0 \\
\beta_{T} T_{c}
\end{array}\right]
$$

where $T_{c}$ is the temperature of the cooling jacket. The rest of parameters take the values (Antoniades and Christofides, 2001):

$$
P e_{C}=7 ; \quad B_{C}=0.1 ; \quad \gamma=10 ; \quad P e_{T}=7 ; \quad B_{T}=2.5 ; \quad \beta_{T}=2 .
$$


The effect of the recycle stream is included in the model through the following boundary conditions:

$$
\begin{gathered}
\nabla z=P e_{z}[r z(t, 1)-z(t, 0)] ; \quad P e_{z}=\left[\begin{array}{cc}
P e_{C} & 0 \\
0 & P e_{T}
\end{array}\right] \quad \text { in } \xi=0 \\
\nabla z=\mathbf{0} ; \quad \text { in } \xi=1
\end{gathered}
$$

where $r$ is the recycle relation.

According to Definition 2, this system is dissipative with respect to the quadratic function $w=\frac{1}{2}\left(C^{2}+T^{2}\right)$. To show this point the limit set $\ell_{0}$, computed by using Eqn (8) in Condition 1, is represented in Figure 5. The continuous black line $(\varpi)$ corresponds with the region where $\ell_{0}=0$, while the area denoted by $\Omega$ depicts the zone where $\ell_{0}<0$. The unfeasible region $\left(C>C_{f}\right)$ is represented

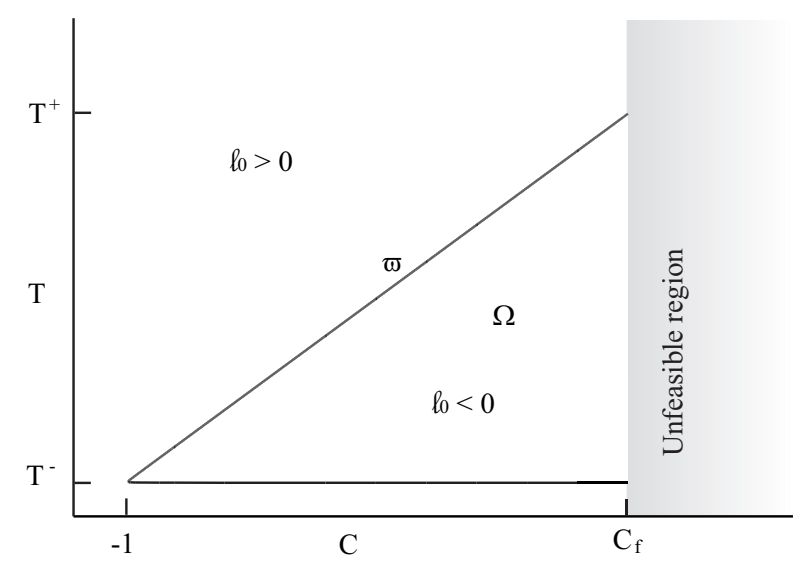

Fig. 5. State space representation of the limit set $\left(\ell_{0}\right)$ for the System (43). The zone where $\ell_{0}=0$ is depicted by the continuous black line $(\varpi)$.

by a gray rectangle. The picture shows that, for a given feed concentration $\left(C_{f}\right)$, there exists an upper temperature $\left(T^{+}\right)$so that, outside the bounded region $\mathcal{D}=\Omega \cup \varpi, \ell_{0}$ is positive.

As mentioned in Section 5 the control law is constructed by using a ROM.

In order to solve the eigenvalue problem (13), a more convenient representation of system (43) with homogeneous boundary conditions will be employed. In that aim, we define the transformation (Balsa-Canto et al., 2004):

$$
W_{z}=z-\chi(\xi) \pi(t) ; \quad \chi(\xi)=\left[\begin{array}{cc}
\chi_{C}(\xi) & 0 \\
0 & \chi_{T}(\xi)
\end{array}\right]
$$


where $W_{z}=\left[W_{C}, W_{T}\right]^{T}$. This transformation is introduced into Eqns (43)-(45) leading to:

$$
\frac{d W_{z}}{d t}+\chi(\xi) \frac{d \pi(t)}{d t}=D \Delta\left(W_{z}+\chi(\xi) \pi(t)\right)-\nabla\left(W_{z}+\chi(\xi) \pi(t)\right)+\Sigma\left(W_{z}\right)+p
$$

with boundary conditions:

$$
\begin{gathered}
\nabla\left(W_{z}+\chi(\xi) \pi(t)\right)=P e_{z}\left(r z(t, 1)-W_{z}(t, 0)-\chi(0) \pi(t)\right) \quad \text { in } \xi=0, \\
\nabla\left(W_{z}+\chi(\xi) \pi(t)\right)=0 \quad \text { in } \xi=1,
\end{gathered}
$$

which become homogeneous by selecting functions $\pi(t)$ and $\chi(\xi)$ of the form:

$$
\pi(t)=z(t, 1) ; \quad \chi_{C}(\xi)=\chi_{T}(\xi)=r ; \forall t, \xi,
$$

so that the system dynamics can be written as:

$$
\frac{d W_{z}}{d t}=D \Delta W_{z}-\nabla W_{z}+\Sigma\left(W_{z}\right)-r \frac{d z(t, 1)}{d t}+p
$$

where

$$
\begin{gathered}
\Sigma\left(W_{z}\right)=\left[\begin{array}{c}
-f\left(W_{C}, W_{T}\right) \\
B_{T} f\left(W_{C}, W_{T}\right)-\beta_{T}\left(W_{T}+r T(t, 1)\right)
\end{array}\right] ; \\
f\left(W_{C}, W_{T}\right)=B_{C}\left(1+W_{C}+r C(t, 1)\right) \exp \left(\frac{\gamma\left(W_{T}+r T(t, 1)\right)}{1+W_{T}+r T(t, 1)}\right),
\end{gathered}
$$

with boundary conditions

$$
\begin{gathered}
\nabla W_{z}=-P e_{z} W_{z} ; \quad \text { in } \xi=0, \\
\nabla W_{z}=0 ; \quad \text { in } \xi=1 .
\end{gathered}
$$

Note that this system match into those described in Section 2. In order to obtain an expression for the term $\frac{d z(t, 1)}{d t}$ we define the operator

$$
P_{m}=\left\{\begin{array}{ll}
0 & \text { if } \xi \neq 1 \\
1 & \text { if } \xi=1
\end{array} .\right.
$$

and project Eqns (46) and (47) over $P_{m}$ resulting on

$$
W_{z}(t, 1)=z(t, 1)-r z(t, 1) \Rightarrow \frac{d W_{z}(t, 1)}{d t}=(1-r) \frac{d z(t, 1)}{d t}
$$

and

$$
\frac{d W_{z}(t, 1)}{d t}=D \Delta W_{z}(t, 1)-\nabla W_{z}(t, 1)+\Sigma\left(W_{z}\right)^{L}-r \frac{d z(t, 1)}{d t}+p^{L},
$$


where $\Sigma\left(W_{z}\right)^{L}$ and $p^{L}$ represent the values of functions $\Sigma\left(W_{z}\right)$ and $p$, respectively, in $\xi=1$. Finally, combining the previous equation with expression (50), leads to:

$$
\frac{d z(t, 1)}{d t}=D \Delta W_{z}(t, 1)-\nabla W_{z}(t, 1)+\Sigma\left(W_{z}\right)^{L}+p^{L} .
$$

The dynamic evolution of system (47) is highly conditioned by the value of the recycle relation. In order to illustrate this point, system (47)-(49) has been numerically solved with different recycle relations. The results for $r=0$ and $r=0.5$ are represented in Figures 6 and 7, respectively. While in the first

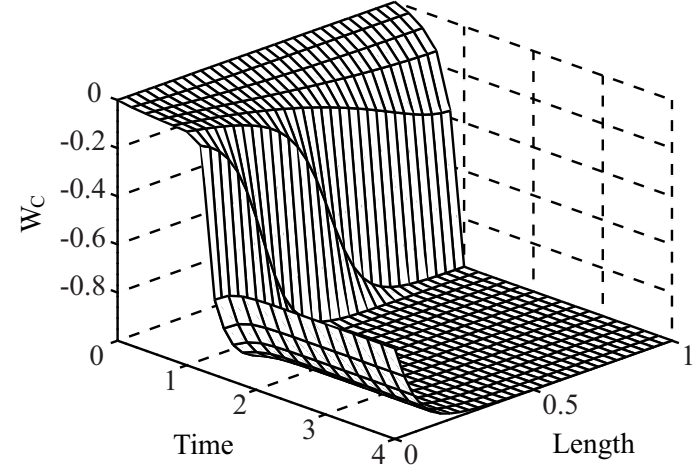

(a)

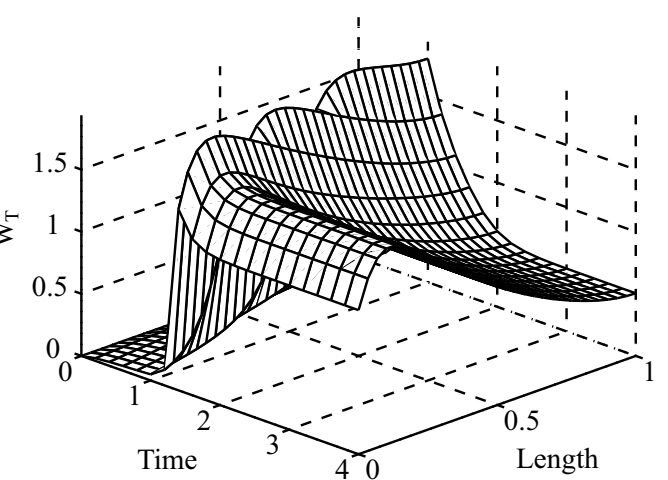

(b)

Fig. 6. Solution of system (47) without recycle $(r=0)$. (a) For the concentration transform $\left(W_{C}\right)$ and (b) for the temperature transform $\left(W_{T}\right)$.

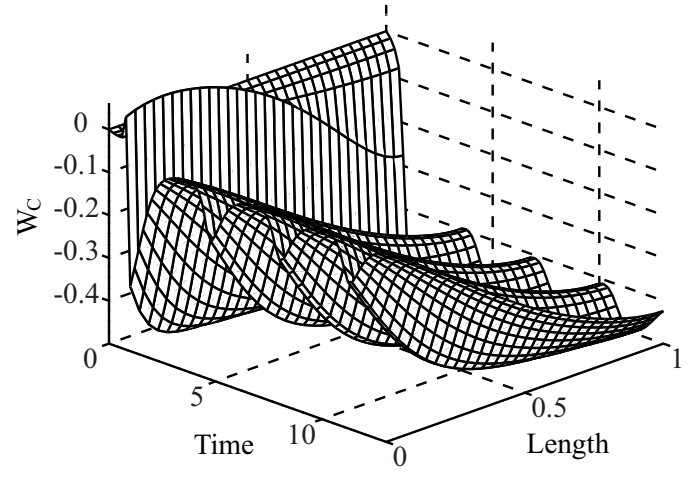

(a)

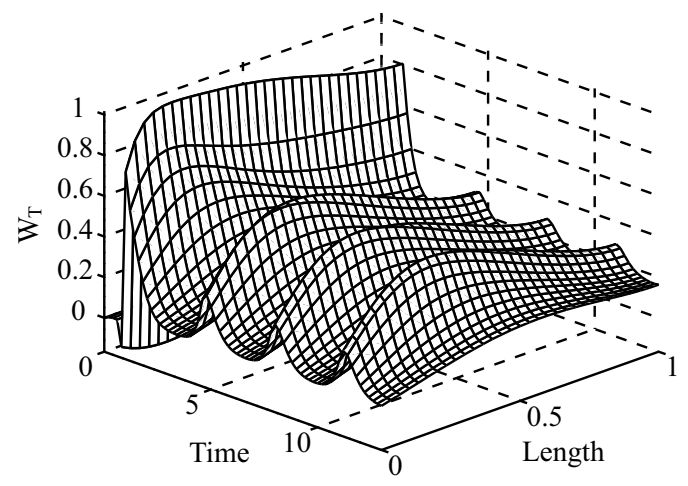

(b)

Fig. 7. Solution of system (47) with recycle $(r=0.5)$. (a) For the concentration transform $\left(W_{C}\right)$ and (b) for the temperature transform $\left(W_{T}\right)$.

case $(r=0)$ a steady state is reached, fixing $r=0.5$ makes the system to evolve to a limit cycle.

The objective of the control law is to produce and to maintain the limit cycle exhibited in Figures 7 (a) and (b) for arbitrary recycle relations (in this case $r=0$ ). In that aim, we have followed the steps described in Section 4 
and employed the ROM of the system exhibiting the limit cycle as the reference trajectory. According to Proposition 1 we define the subsets $\left(S_{a}, \Lambda_{a}, \mathcal{N}_{a}\right)$ and $\left(S_{b}, \Lambda_{b}, \mathcal{N}_{b}\right)$. Then, we project the reference trajectory and the system to be controlled over these subsets. Finally, the results of these projections are subtracted so as to obtain:

$$
\begin{aligned}
\frac{d \bar{m}_{W_{T a}}}{d t}=\frac{1}{P e_{T}}\left\langle\Phi_{a}, \Delta \bar{W}_{T}\right\rangle_{\mathscr{V}} & -\left\langle\Phi_{a}, \nabla \bar{W}_{T}\right\rangle_{\mathscr{V}}+\left\langle\Phi_{a}, \bar{F}\right\rangle_{\mathscr{V}}-\left\langle\Phi_{a}, \beta_{T} \bar{W}_{T}\right\rangle_{\mathscr{V}}+ \\
& \beta_{T}\left\langle\Phi_{a}, \bar{T}_{c}\right\rangle_{\mathscr{V}}, \\
\frac{d \bar{m}_{W_{T b}}}{d t}=\frac{1}{P e_{T}}\left\langle\Phi_{b}, \Delta \bar{W}_{T}\right\rangle_{\mathscr{V}} & -\left\langle\Phi_{b}, \nabla \bar{W}_{T}\right\rangle_{\mathscr{V}}+\left\langle\Phi_{b}, \bar{F}\right\rangle_{\mathscr{V}}-\left\langle\Phi_{b}, \beta_{T} \bar{W}_{T}\right\rangle_{\mathscr{V}} \\
& +\beta_{T}\left\langle\Phi_{b}, \bar{T}_{c}\right\rangle_{\mathscr{V}},
\end{aligned}
$$

where $\bar{F}=B_{T}\left(f-f^{*}\right)-r\left(\frac{d \bar{T}(t, 1)}{d t}+\beta_{T} \bar{T}(t, 1)\right)$. Note that this system is a particular case of Eqns (32) and (33). The main difference is that now, parametric uncertainties are not considered. Adapting control laws (37) and (39) to this case we have that $\bar{T}_{c}=\bar{p}_{a}+\bar{p}_{b}$ with

$$
\begin{aligned}
& \bar{p}_{a}=\left\{\begin{array}{lll}
-\frac{1}{\beta_{T}} \omega_{a}^{*} \frac{\bar{W}_{T a}}{\left\|\bar{W}_{T a}\right\|_{\mathscr{V}}} & \text { if } & \omega_{a}^{*}\left\|\bar{W}_{T a}\right\|_{\mathscr{V}} \geq \theta_{a} \\
-\frac{1}{\beta_{T}}\left(\omega_{a}^{*}\right)^{2} \frac{\bar{W}_{T a}}{\theta_{a}} & \text { if } & \omega_{a}^{*}\left\|\bar{W}_{T a}\right\|_{\mathscr{V}}<\theta_{a}
\end{array}\right. \\
& \bar{p}_{b}=\left\{\begin{array}{lll}
-\frac{1}{\beta_{T}} \omega_{b}^{*} \frac{\bar{W}_{T b}}{\left\|\bar{W}_{T b}\right\|_{\mathscr{V}}} & \text { if } & \omega_{b}^{*}\left\|\bar{W}_{T b}\right\|_{\mathscr{V}} \geq \theta_{b} \\
-\frac{1}{\beta_{T}}\left(\omega_{b}^{*}\right)^{2} \frac{\bar{W}_{T b}}{\theta_{b}} & \text { if } & \omega_{b}^{*}\left\|\bar{W}_{T b}\right\|_{\mathscr{V}}<\theta_{b}
\end{array}\right.
\end{aligned}
$$

where $\omega_{a}^{*}=\omega_{b}^{*}=\max _{\mathscr{V}}(\bar{F}) \geq \bar{F}$. The application of this control to the previous system will make the field $z$ to evolve to a region arbitrarily close to the limit cycle $z^{*}$. The fields $\bar{W}_{T a}$ and $\bar{W}_{T b}$ are calculated from the measurements of the field as:

$$
\bar{W}_{T a}=\Phi_{a}\left\langle\Phi_{a}, \bar{W}_{T}\right\rangle ; \quad \bar{W}_{T b}=\bar{W}_{T}-\bar{W}_{T a} .
$$

It is pointed out that using a ROM with 8 representative modes, results into a good approximation to the reference trajectory behavior. On the other hand, the selection of a restrictive bound on the nonlinear term and the choice of small parameters $\theta_{a}$ and $\theta_{b}$ may result in large control efforts. In order to avoid this problem, we impose the following bounds on the control actions: $-2.5<\bar{T}_{c}<2.5$. Figures 8 (a) and (b) show the evolution of system (47) under control and the control effort $\left(\left\|\bar{T}_{c}\right\|_{\mathscr{V}}\right)$, respectively. As shown in Figure 8 (a), the system begins to behave as in the case with recycle $(r=0)$. When the control law enters in action $(t=3)$, the system reaches the reference trajectory after a short transition period. 


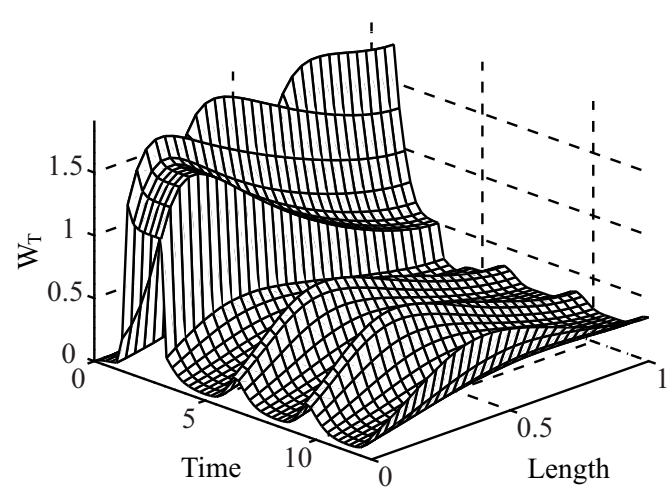

(a)

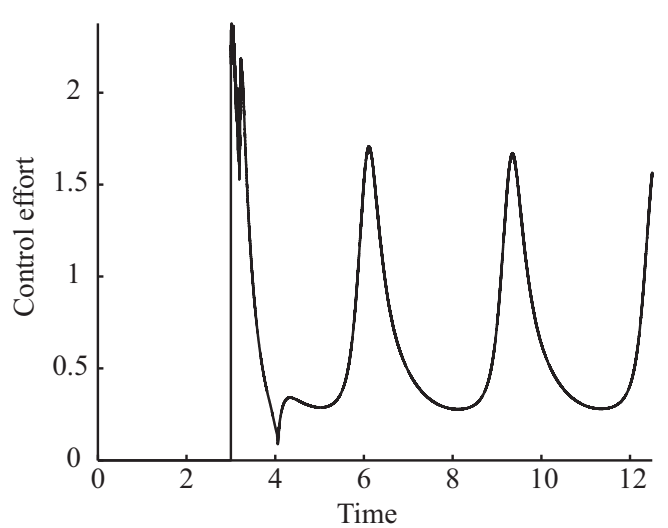

(b)

Fig. 8. (a) Effect of the control laws (51) and (52) on the field $W_{T}$. (b) Control effort.

\section{Conclusions}

In this work, a class of controllers able to force a chemical distributed system to follow stationary or time dependent reference trajectories has been proposed. The design methodology combine classical results on the nonlinear control of finite dimensional systems with reduction order techniques. The control law is able to stabilize the reference even when detailed information on the system parameters or in the structure of the nonlinear terms is not available. In this context, the Kirchhoff transform was shown to be a useful tool to deal with the nonlinearity associated with diffusion term and to construct the ROM representation. Such representation is a key element in the derivation of the controller logic as well as in the stability analysis of the control law. Regarding the problem of controller saturation, it has been shown that the use of the available information on the system structure might have an important effect on the control effort. In addition, problems related to systems with inhomogeneous boundary conditions were efficiently handled by means of a systematic approach, which consisted of the definition of given boundary transformations. Finally, the stability and robustness properties of the control were illustrated on a number of computational experiments concerning tubular reactors. 


\section{Notation}

A Intensive variables

a Convex function

$\mathscr{B} \quad$ Boundary of the spatial domain

C Concentration

$\mathscr{D} \quad$ Spatial and time domains

L Field dependent diffusion matrix

m Modes of the field

n Unit normal pointing outward from a boundary element

ns Number of states

p Control terms

$\mathfrak{p} \quad$ Manipulated input

Pe Peclet number

r Recycle relation

s Entropy

t Time

T Temperature

$\mathscr{T}$ Time domain

v Velocity field

$\mathscr{V} \quad$ Spatial domain

$w \quad$ Positive convex function

$\mathcal{W}$ Spatial integral of $w$

$\mathrm{X}$ Microscopic fluxes through the domain

y Measured output

z Extensive variables

Greek symbols

$\Gamma \quad$ Kirchhoff transform

$\lambda \quad$ Eigenvalue

$\phi \quad$ Eigenfunction 
$\xi \quad$ Spatial coordinates

$\Sigma \quad$ Nonlinear terms

Subindex

a Finite dimensional set

b Infinite dimensional set

Superindex

* Reference

T Transpose

Mathematical operators

$\nabla \quad$ Gradient operator

$\Delta \quad$ Laplacian operator

$\langle f, g\rangle_{\mathscr{V}} \quad$ Inner product of two vector functions: $\langle f, g\rangle_{\mathscr{V}}=\int_{\mathscr{V}} f^{T} g d \xi$

$\|f\|_{\mathscr{V}} \quad \mathcal{L}_{2}$ norm defined as $\|f\|_{\mathscr{V}}=\langle f, f\rangle_{\mathscr{V}}^{1 / 2}$

Abbreviations

ODE Ordinary differential equation

PDE Partial differential equation

POD Proper orthogonal decomposition

RD Reaction-Diffusion

RDC Reaction-Diffusion-Convection

RHS Right hand side

ROM Reduced order model

\section{Acknowledgements}

The authors acknowledge financial support received from the Spanish Government (MCyT Project DPI2004-07444-C04-03) and Xunta de Galicia (PGIDIT02PXIC40209PN). 


\section{Appendices}

A

Lemma 1 Under Condition 1, system (7) is passive with respect to an output $y=\bar{A}$ and an input $\mathfrak{p}=\bar{p}+\mu y$.

\section{B Proof of Lemma 1}

Using the function $a(z)$ of Section 2, we define a new convex function constructed as the difference between the original $a(z)$ and its supporting hyperplane at the reference $z^{*}$ :

$$
w\left(z ; z^{*}\right)=a(z)-\left[a\left(z^{*}\right)+\left(z-z^{*}\right)^{T} A^{*}\right] \geq 0 .
$$

Time derivative of $w\left(z ; z^{*}\right)$ combined with Eqns (7) and (8) leads to:

$$
\frac{d w}{d t}=\frac{d w^{T}}{d \bar{z}} \frac{\partial \bar{z}}{\partial t}=\bar{A}^{T} \Delta \bar{\Gamma}-\bar{A}^{T} \nabla(\mathbf{v} \bar{z})-\ell_{\mu}+\mu \bar{A}^{T} \bar{A}+\bar{A}^{T} \bar{p}
$$

note that now $\bar{A}$ is defined from the convex function $w$ as $\bar{A}=\frac{\partial w}{\partial \bar{z}}$. Choosing the output and the input as indicated in Lemma 1, integrating expression (B.2) over the spatial domain and denoting $\mathcal{W}=\int_{\mathscr{V}} w\left(z ; z^{*}\right) d \xi$ and $L_{\mu}=$ $\int_{\mathscr{V}} \ell_{\mu}\left(z ; z^{*}\right) d \xi$, we have:

$$
\frac{d \mathcal{W}}{d t}=\langle\bar{A}, \Delta \bar{\Gamma}\rangle_{\mathscr{V}}-\langle\bar{A}, \nabla(\mathbf{v} \bar{z})\rangle_{\mathscr{V}}-L_{\mu}+\langle y, \mathfrak{p}\rangle_{\mathscr{V}}
$$

Applying the Green's formula to the first term of the right hand side (RHS) of equation (B.3):

$$
\langle\bar{A}, \Delta \bar{\Gamma}\rangle_{\mathscr{V}}=\int_{\mathscr{B}} \bar{A}^{T} L(A) \frac{d \bar{A}}{d \mathbf{n}} d \xi-\langle\nabla \bar{A}, L(A) \nabla \bar{A}\rangle_{\mathscr{V}}
$$

due to boundary conditions (9) and (10) the first term of the RHS is non positive. Since $L(A)$ is positive definite the second term of the RHS is non negative then $\langle\bar{A}, \Delta \bar{\Gamma}\rangle_{\mathscr{V}} \leq 0$. Consider now the second term of the RHS of Eqn (B.3) with the velocity term (v) constant:

$$
\begin{aligned}
-\langle\bar{A}, \nabla(\mathbf{v} \bar{z})\rangle_{\mathscr{V}}=-\int_{\mathscr{V}} & \left(\frac{\partial w}{\partial \bar{z}}\right)^{T} \nabla(\mathbf{v} \bar{z}) d \xi=-\int_{\mathscr{V}} \nabla\left(\mathbf{v} w\left(z, z^{*}\right)\right) d \xi= \\
& -\int_{\mathscr{B}} \mathbf{v n} w\left(z, z^{*}\right) d \xi
\end{aligned}
$$


In the part of the boundary where the fluid enters it follows that $z=z^{*}$ - see Eqn (11)-, so according to (B.1) $\left.w\right|_{\mathscr{B}_{c}^{-}}=0$. In addition, in $\mathscr{B}_{c}^{+}$it follows that vn $\geq 0$ and since $w\left(z, z^{*}\right)$ is non negative, we get:

$$
-\int_{\mathscr{B}} \mathbf{v n} w\left(z, z^{*}\right) d \xi=-\int_{\mathscr{B}_{c}^{+}} \mathbf{v n} w\left(z, z^{*}\right) d \xi \leq 0 .
$$

Now, from Condition 1, we have that there exists a positive constant $\mu$ so that $L_{\mu}>0$. Then equation (B.3) can be rewritten as:

$$
\frac{d \mathcal{W}}{d t} \leq\langle y, \mathfrak{p}\rangle_{\mathscr{V}}
$$

and the result follows by integrating this inequality over the time interval $(t, t+T)$.

C

Lemma 2 Projections of the different terms of Eqn (7) over the subfields $\bar{A}_{b}$ and $\bar{A}_{a}$ satisfy the following relations:

(1) $\left\langle\bar{A}_{b}, \frac{\partial \bar{z}}{\partial t}\right\rangle_{\mathscr{V}}=\alpha_{b}^{T} \frac{d m_{b}}{d t}$

(2) $\left\langle\bar{A}_{b}, \Delta \bar{\Gamma}\right\rangle_{\mathscr{V}} \leq-\zeta \lambda_{\ell} \alpha_{b}^{T} \alpha_{b}$

(3) $-\left\langle\bar{A}_{b}, \nabla(\boldsymbol{v} \bar{z})\right\rangle_{\mathscr{V}} \leq 0$

(4) $\left\langle\bar{A}_{b}, \bar{\Sigma}\right\rangle_{\mathscr{V}} \leq \mu \alpha_{b}^{T} \alpha_{b}$

(5) $\left\langle\bar{A}_{a}, \Delta \bar{\Gamma}\right\rangle_{\mathscr{V}} \leq-\zeta \lambda_{m} \alpha_{a}^{T} \alpha_{a}$

(6) $-\left\langle\bar{A}_{a}, \Delta \Gamma_{d}\right\rangle_{\mathscr{V}} \leq \lambda_{q}\left\|\gamma_{d a}\right\|_{2}\left\|\bar{A}_{a}\right\|_{\mathscr{V}}$

(7) $-\left\langle\bar{A}_{a}, \nabla(\boldsymbol{v} \bar{z})\right\rangle_{\mathscr{V}} \leq 0$

(8) $\left\langle\bar{A}_{a}, \bar{\Sigma}\right\rangle_{\mathscr{V}} \leq \eta_{a}\left\|\bar{A}_{a}\right\|_{\mathscr{V}}$

where $\alpha_{b}=\left[\alpha_{\mathcal{N}_{b}(1)}, \alpha_{\mathcal{N}_{b}(2)}, \ldots\right]^{T}, m_{b}=\left[m_{\mathcal{N}_{b}(1)}, m_{\mathcal{N}_{b}(2)}, \ldots\right]^{T}, \lambda_{\ell}=\min _{\lambda}\left(\Lambda_{b}\right)$, $\zeta=\min _{\bar{A}}\left(\inf _{i}\left[\lambda_{i}(L)\right]\right), \alpha_{a}=\left[\alpha_{\mathcal{N}_{a}(1)}, \alpha_{\mathcal{N}_{a}(2)}, \ldots, \alpha_{\mathcal{N}_{a}(n)}\right]^{T}, \lambda_{m}=\min _{\lambda}\left(\Lambda_{a}\right), \lambda_{q}=$ $\max _{\lambda}\left(\Lambda_{a}\right)$, and $\eta_{a} \geq\left\|\bar{\Sigma}_{a}\right\|_{\mathscr{V}}$.

\section{Proof of Lemma 2}

(1) The first statement of the Lemma is proved by using the separation property of the field and its dual and the orthonormality of eigenfunctions:

$$
\left\langle\bar{A}_{b}, \frac{\partial \bar{z}}{\partial t}\right\rangle_{\mathscr{V}}=\left\langle\sum_{i \in \mathcal{N}_{b}} \alpha_{i} \phi_{i}, \sum_{i \in \mathbb{N}} \frac{d m_{i}}{d t} \phi_{i}\right\rangle_{\mathscr{V}}=\sum_{i \in \mathcal{N}_{b}} \alpha_{i} \frac{d m_{i}}{d t} \Rightarrow
$$




$$
\left\langle\bar{A}_{b}, \frac{\partial \bar{z}}{\partial t}\right\rangle_{\mathscr{V}}=\alpha_{b}^{T} \frac{d m_{b}}{d t} .
$$

(2) The proof of the second point is more complicated and begins by expanding the field $\bar{A}$ and the Kirchhoff transform $\bar{\Gamma}$ as in (23) and (26) respectively:

$$
\begin{gathered}
\left\langle\bar{A}_{b}, \Delta \bar{\Gamma}\right\rangle_{\mathscr{V}}= \\
\left\langle\sum_{i \in \mathcal{N}_{b}} \alpha_{i} \phi_{i}, \sum_{i \in \mathbb{N}} \gamma_{i} \Delta \phi_{i}\right\rangle_{\mathscr{V}}=\left\langle\sum_{i \in \mathcal{N}_{b}} \alpha_{i} \phi_{i}, \sum_{i \in \mathbb{N}}-\gamma_{i} \lambda_{i} \phi_{i}\right\rangle_{\mathscr{V}}= \\
\left\langle\sum_{i \in \mathcal{N}_{b}} \alpha_{i} \phi_{i}, \sum_{i \in \mathcal{N}_{b}}-\gamma_{i} \lambda_{i} \phi_{i}\right\rangle_{\mathscr{V}}=\left\langle\bar{A}_{b}, \Delta \bar{\Gamma}_{b}\right\rangle_{\mathscr{V}},
\end{gathered}
$$

now, using the divergence theorem we have that:

$$
\begin{gathered}
\int_{\mathscr{V}} \nabla\left(\bar{A}_{b}^{T} \nabla \bar{\Gamma}_{b}\right) d \xi=\left\langle\nabla \bar{A}_{b}, \nabla \bar{\Gamma}_{b}\right\rangle_{\mathscr{V}}+\left\langle\bar{A}_{b}, \Delta \bar{\Gamma}_{b}\right\rangle_{\mathscr{V}} \Rightarrow \int_{\mathscr{B}} \bar{A}_{b}^{T} \frac{d \bar{\Gamma}_{b}}{d \mathbf{n}} d \xi= \\
\left\langle\nabla \bar{A}_{b}, \nabla \bar{\Gamma}_{b}\right\rangle_{\mathscr{V}}+\left\langle\bar{A}_{b}, \Delta \bar{\Gamma}_{b}\right\rangle_{\mathscr{V}}=\left\langle\bar{A}_{b}, L(A) \nabla \bar{A}_{b}\right\rangle_{\mathscr{V}}+\left\langle\bar{A}_{b}, \Delta \bar{\Gamma}_{b}\right\rangle_{\mathscr{V}}
\end{gathered}
$$

rearranging the terms and denoting $\zeta=\min _{\bar{A}}\left(\inf _{i}\left[\lambda_{i}(L)\right]\right)>0$, it is obtained:

$$
\left\langle\bar{A}_{b}, \Delta \bar{\Gamma}_{b}\right\rangle_{\mathscr{V}} \leq \int_{\mathscr{B}} \bar{A}_{b}^{T} L(A) \frac{d \bar{A}_{b}}{d \mathbf{n}} d \xi-\zeta\left\langle\nabla \bar{A}_{b}, \nabla \bar{A}_{b}\right\rangle_{\mathscr{V}} .
$$

On the other hand, it follows that:

$$
\begin{gathered}
\zeta \int_{\mathscr{V}} \nabla\left(\bar{A}_{b}^{T} \nabla \bar{A}_{b}\right) d \xi=\zeta\left\langle\nabla \bar{A}_{b}, \nabla \bar{A}_{b}\right\rangle_{\mathscr{V}}+\zeta\left\langle\bar{A}_{b}, \Delta \bar{A}_{b}\right\rangle_{\mathscr{V}} \Rightarrow \\
\zeta\left\langle\nabla \bar{A}_{b}, \nabla \bar{A}_{b}\right\rangle_{\mathscr{V}}=\zeta \int_{\mathscr{B}} \bar{A}_{b}^{T} \frac{d \bar{A}_{b}}{d \mathbf{n}} d \xi-\zeta\left\langle\bar{A}_{b}, \Delta \bar{A}_{b}\right\rangle_{\mathscr{V}} .
\end{gathered}
$$

Substituting equation (D.2) on inequality (D.1):

$$
\left\langle\bar{A}_{b}, \Delta \bar{\Gamma}_{b}\right\rangle_{\mathscr{V}} \leq \int_{\mathscr{B}} \bar{A}_{b}^{T} L(A) \frac{d \bar{A}_{b}}{d \mathbf{n}} d \xi-\zeta \int_{\mathscr{B}} \bar{A}_{b}^{T} \frac{d \bar{A}_{b}}{d \mathbf{n}} d \xi+\zeta\left\langle\bar{A}_{b}, \Delta \bar{A}_{b}\right\rangle_{\mathscr{V}} .
$$

Boundary conditions (9) and (10) make the term $\left(\int_{\mathscr{B}} \bar{A}_{b}^{T} L(A) \frac{d \bar{A}_{b}}{d \mathbf{n}} d \xi-\right.$ $\left.\zeta \int_{\mathscr{B}} \bar{A}_{b}^{T} \frac{d \bar{A}_{b}}{d \mathbf{n}} d \xi\right)$ to be non positive, then it follows that

$$
\begin{gathered}
\left\langle\bar{A}_{b}, \Delta \bar{\Gamma}_{b}\right\rangle_{\mathscr{V}} \leq \zeta\left\langle\bar{A}_{b}, \Delta \bar{A}_{b}\right\rangle_{\mathscr{V}}=\zeta\left\langle\sum_{i \in \mathcal{N}_{b}} \alpha_{i} \phi_{i}, \sum_{i \in \mathcal{N}_{b}}-\alpha_{i} \lambda_{i} \phi_{i}\right\rangle_{\mathscr{V}}= \\
\zeta \sum_{i \in \mathcal{N}_{b}}-\lambda_{i} \alpha_{i}^{2}=-\zeta \alpha_{b}^{T} \Upsilon_{b} \alpha_{b},
\end{gathered}
$$

where $\Upsilon_{b}$ is a diagonal matrix containing the eigenvalues of $\left(\Lambda_{b}\right)$. And the result follows by choosing $\lambda_{\ell}=\min _{\lambda}\left(\Lambda_{b}\right)$. 
(3) Applying the series expansion to the terms of the third statement of Lemma 2, we obtain:

$$
\begin{gathered}
-\left\langle\bar{A}_{b}, \nabla(\mathbf{v} \bar{z})\right\rangle_{\mathscr{V}}=-\left\langle\sum_{i \in \mathcal{N}_{b}} \alpha_{i} \phi_{i}, \mathbf{v} \sum_{i \in \mathbb{N}} \tau_{i} \phi_{i}\right\rangle_{\mathscr{V}}=-\left\langle\sum_{i \in \mathcal{N}_{b}} \alpha_{i} \phi_{i}, \mathbf{v} \sum_{i \in \mathcal{N}_{b}} \tau_{i} \phi_{i}\right\rangle_{\mathscr{V}}= \\
-\left\langle\bar{A}_{b}, \nabla\left(\mathbf{v} \bar{z}_{b}\right)\right\rangle_{\mathscr{V}}
\end{gathered}
$$

Now, defining a new convex function $w_{b}$ of the form of (B.1) for the field $\bar{z}_{b}$

$$
\begin{gathered}
\bar{A}_{b}^{T} \nabla\left(\mathbf{v} \bar{z}_{b}\right)=\frac{\partial\left(\mathbf{v} w_{b}\right)}{\partial\left(\mathbf{v} \bar{z}_{b}\right)} \nabla\left(\mathbf{v} \bar{z}_{b}\right)=\nabla\left(\mathbf{v} w_{b}\right) \Rightarrow-\left\langle\bar{A}_{b}, \nabla\left(\mathbf{v} \bar{z}_{b}\right)\right\rangle_{\mathscr{V}}= \\
\int_{\mathscr{V}} \nabla\left(\mathbf{v} w_{b}\right) d \xi=\int_{\mathscr{B}_{c}^{+}} \mathbf{n v} w_{b} d \xi,
\end{gathered}
$$

and using the boundary conditions, results:

$$
-\left\langle\bar{A}_{b}, \nabla(\mathbf{v} \bar{z})\right\rangle_{\mathscr{V}} \leq 0
$$

(4) In the proof of the fourth point we will make use of the integral version of equation (8):

$$
\langle\bar{A}, \bar{\Sigma}\rangle_{\mathscr{V}}+L_{\mu}=\mu\langle\bar{A}, \bar{A}\rangle_{\mathscr{V}}
$$

On the other hand, making use of the series expansion we have that

$$
\langle\bar{A}, \bar{\Sigma}\rangle_{\mathscr{V}}=\sum_{i \in \mathbb{N}} \alpha_{i} \sigma_{i} ; \quad\langle\bar{A}, \bar{A}\rangle_{\mathscr{V}}=\sum_{i \in \mathbb{N}} \alpha_{i}^{2}
$$

Substituting these expressions in (D.3) we obtain, for a given $\mu>0$,

$$
\sum_{i \in \mathbb{N}}\left(\mu \alpha_{i}^{2}-\alpha_{i} \sigma_{i}\right)=L_{\mu} \geq 0
$$

In order for this inequality to hold for any field satisfying (22) we need $\mu \alpha_{k}^{2}-\alpha_{k} \sigma_{k}>0$ for all $k$. This argument allows us to construct expressions equivalent to (D.3) for each subfield $\bar{A}_{a}$ and $\bar{A}_{b}$ and for the nonlinear terms $\bar{\Sigma}_{a}$ and $\bar{\Sigma}_{b}$. In particular, we have that

$$
\left\langle\bar{A}_{b}, \bar{\Sigma}_{b}\right\rangle_{\mathscr{V}}+L_{\mu}=\mu\left\langle\bar{A}_{b}, \bar{A}_{b}\right\rangle_{\mathscr{V}}
$$

Using (25) and the orthonormality condition we obtain $\left\langle\bar{A}_{b}, \bar{\Sigma}\right\rangle_{\mathscr{V}}=\left\langle\bar{A}_{b}, \bar{\Sigma}_{b}\right\rangle_{\mathscr{V}}$ so it follows that

$$
\left\langle\bar{A}_{b}, \bar{\Sigma}\right\rangle_{\mathscr{V}} \leq \mu\left\langle\bar{A}_{b}, \bar{A}_{b}\right\rangle_{\mathscr{V}}=\mu \alpha_{b}^{T} \alpha_{b}
$$

(5) The steps to be followed in the proof of the fifth statement are similar to those followed in the proof of the second point for the term $\left\langle\bar{A}_{b}, \Delta \bar{\Gamma}\right\rangle_{\mathscr{V}}$ so they will not be included in this document. 
(6) In order to prove the sixth statement we make again use of the series expansion:

$$
\begin{gathered}
-\left\langle\bar{A}_{a}, \Delta \Gamma_{d}\right\rangle_{\mathscr{V}}=-\left\langle\sum_{i \in \mathcal{N}_{a}} \alpha_{i} \phi_{i}, \sum_{i \in \mathbb{N}}-\lambda_{i} \gamma_{d i} \phi_{i}\right\rangle_{\mathscr{V}}=\sum_{i \in \mathcal{N}_{a}} \lambda_{i} \alpha_{i} \gamma_{d i} \leq \\
\lambda_{q} \sum_{i \in \mathcal{N}_{a}}\left|\alpha_{i}\right|\left|\gamma_{d i}\right|,
\end{gathered}
$$

where $\lambda_{q}=\max _{\lambda} \Lambda_{a}$. Using the Hölder inequality

$$
-\left\langle\bar{A}_{a}, \Delta \Gamma_{d}\right\rangle_{\mathscr{V}} \leq \lambda_{q}\left\|\alpha_{a}\right\|_{2}\left\|\gamma_{d a}\right\|_{2}=\lambda_{q}\left\|\gamma_{d a}\right\|_{2}\left\|\bar{A}_{a}\right\|_{\mathscr{V}}
$$

(7) This proof is similar to the one of point 3 :

$$
\begin{gathered}
-\left\langle\bar{A}_{a}, \nabla(\mathbf{v} \bar{z})\right\rangle_{\mathscr{V}}=-\left\langle\sum_{i \in \mathcal{N}_{a}} \alpha_{i} \phi_{i}, \mathbf{v} \sum_{i \in \mathbb{N}} \tau_{i} \phi_{i}\right\rangle_{\mathscr{V}}=-\left\langle\sum_{i \in \mathcal{N}_{a}} \alpha_{i} \phi_{i}, \mathbf{v} \sum_{i \in \mathcal{N}_{a}} \tau_{i} \phi_{i}\right\rangle_{\mathscr{V}}= \\
-\left\langle\bar{A}_{a}, \nabla\left(\mathbf{v} \bar{z}_{a}\right)\right\rangle_{\mathscr{V}} .
\end{gathered}
$$

Defining a new convex function $w_{a}$ of the form of (B.1) for the field $\bar{z}_{a}$

$$
\bar{A}_{a}^{T} \nabla\left(\mathbf{v} \bar{z}_{a}\right)=\nabla\left(\mathbf{v} w_{a}\right) \Rightarrow-\left\langle\bar{A}_{a}, \nabla\left(\mathbf{v} \bar{z}_{a}\right)\right\rangle_{\mathscr{V}}=\int_{\mathscr{V}} \nabla\left(\mathbf{v} w_{a}\right) d \xi=\int_{\mathscr{B}_{c}^{+}} \mathbf{n v} w_{a} d \xi
$$

and the result follows by using the boundary conditions.

(8) The last statement is proved by using a known bound on the nonlinear term $\eta_{a} \geq\left\|\bar{\Sigma}_{a}\right\|_{\mathscr{V}}$, so by means of the Schwartz inequality

$$
\left\langle\bar{A}_{a}, \bar{\Sigma}\right\rangle_{\mathscr{V}} \leq\left\|\bar{A}_{a}\right\|_{\mathscr{V}}\|\bar{\Sigma}\|_{\mathscr{V}} \leq \eta_{a}\left\|\bar{A}_{a}\right\|_{\mathscr{V}}
$$

\section{E Proof of Proposition 1}

The projection of system (7) over the subfield $\bar{A}_{b}$, with $\bar{p}=0$, leads to:

$$
\left\langle\bar{A}_{b}, \frac{\partial \bar{z}}{\partial t}\right\rangle_{\mathscr{V}}=\left\langle\bar{A}_{b}, \Delta \bar{\Gamma}\right\rangle_{\mathscr{V}}-\left\langle\bar{A}_{b}, \nabla(\mathbf{v} \bar{z})\right\rangle_{\mathscr{V}}+\left\langle\bar{A}_{b}, \bar{\Sigma}\right\rangle_{\mathscr{V}}
$$

Making use of relations (1)-(4) of Lemma 2, Eqn. (E.1) can be rewritten as:

$$
\alpha_{b}^{T} \frac{d m_{b}}{d t} \leq\left(\mu-\zeta \lambda_{\ell}\right) \alpha_{b}^{T} \alpha_{b}
$$

A new convex function $w_{b}$ is defined as in Eqn (B.1) for the field $\bar{z}_{b}$. The time derivative of $w_{b}$ yields $\frac{d\left(w_{b}\right)}{d t}=\bar{A}_{b}^{T} \frac{\partial \bar{z}_{b}}{\partial t}$. Integrating this expression over the spatial domain, results into

$$
\int_{\mathscr{V}} \frac{d w_{b}}{d t} d \xi=\frac{d \mathcal{W}_{b}}{d t}=\left\langle\bar{A}_{b}, \frac{\partial \bar{z}}{\partial t}\right\rangle_{\mathscr{V}}=\sum_{i \in \mathcal{N}_{b}} \alpha_{i} \frac{d m_{i}}{d t}=\alpha_{b}^{T} \frac{d m_{b}}{d t} .
$$


On the other hand, we know from (17) that function $\mathcal{W}_{b}$ is bounded by the field $\bar{z}_{b}$ as:

$$
q_{0}\left\|\bar{z}_{b}\right\|_{\mathscr{V}}^{2} \leq \mathcal{W}_{b} \leq q_{1}\left\|\bar{z}_{b}\right\|_{\mathscr{V}}^{2}=q_{1} m_{b}^{T} m_{b}
$$

and since $\bar{A}_{b}$ relates to $\bar{z}_{b}$ through an expression of the form (2), we have:

$$
\alpha_{b}^{T} \alpha_{b}=\left\langle\bar{A}_{b}, \bar{A}_{b}\right\rangle_{\mathscr{V}}=\left\langle Q \bar{z}_{b}, Q \bar{z}_{b}\right\rangle_{\mathscr{V}} \geq \delta_{0}^{2} m_{b}^{T} m_{b} \geq \frac{\delta_{0}^{2}}{q_{1}} \mathcal{W}_{b}
$$

where $\delta_{0}$ is the minimum eigenvalue of $Q$. Substituting (E.3) and (E.4) in (E.2), we obtain

$$
\frac{d \mathcal{W}_{b}}{d t} \leq\left(\mu-\zeta \lambda_{\ell}\right) \frac{\delta_{0}^{2}}{q_{1}} \mathcal{W}_{b}
$$

Since the eigenvalues have the property that $\lambda_{i} \rightarrow \infty$ as $i \rightarrow \infty$, we can always find a large enough number $n$ of elements in the set $\left(S_{a}, \Lambda_{a}, \mathcal{N}_{a}\right)$ so that $\left(\mu-\zeta \lambda_{\ell}\right)<0$. For this number $n$ and by means of the Gronwall-Bellman theorem, it follows that $\mathcal{W}_{b}$, and thus $\bar{z}_{b}$, converge exponentially to zero.

\section{F $\quad$ Proof of Proposition 2}

Substituting the control law on inequality (36), four cases arise:

- If $\omega_{a}\left\|\bar{A}_{a}\right\|_{\mathscr{V}} \geq \theta_{a}$ and $\omega_{a}^{*}\left\|\bar{A}_{a}\right\|_{\mathscr{V}} \geq \theta_{a}$ then

$$
\begin{gathered}
\frac{d \mathcal{W}_{a}}{d t} \leq-\zeta \lambda_{m} \frac{\delta_{0}^{2}}{q_{1}} \mathcal{W}_{a}+\lambda_{q}\left\|\gamma_{d a}\right\|_{2}\left\|\bar{A}_{a}\right\|_{\mathscr{V}}+\eta_{a}\left\|\bar{A}_{a}\right\|_{\mathscr{V}}-\omega_{a}\left\|\bar{A}_{a}\right\|_{\mathscr{V}}-\omega_{a}^{*}\left\|\bar{A}_{a}\right\|_{\mathscr{V}} \leq \\
-\zeta \lambda_{m} \frac{\delta_{0}^{2}}{q_{1}} \mathcal{W}_{a},
\end{gathered}
$$

so by means of Gronwall-Bellman theorem (Khalil, 1996) $\mathcal{W}_{a}$, and then $\bar{z}_{a}$, tend to zero exponentially fast.

- If $\omega_{a}\left\|\bar{A}_{a}\right\|_{\mathscr{V}} \geq \theta_{a}$ and $\omega_{a}^{*}\left\|\bar{A}_{a}\right\|_{\mathscr{V}}<\theta_{a}$ then

$$
\begin{gathered}
\frac{d \mathcal{W}_{a}}{d t} \leq-\zeta \lambda_{m} \frac{\delta_{0}^{2}}{q_{1}} \mathcal{W}_{a}+\lambda_{q}\left\|\gamma_{d a}\right\|_{2}\left\|\bar{A}_{a}\right\|_{\mathscr{V}}+\eta_{a}\left\|\bar{A}_{a}\right\|_{\mathscr{V}}-\omega_{a}\left\|\bar{A}_{a}\right\|_{\mathscr{V}}-\frac{\left(\omega_{a}^{*}\right)^{2}}{\theta_{a}}\left\|\bar{A}_{a}\right\|_{\mathscr{V}}^{2} \leq \\
-\zeta \lambda_{m} \frac{\delta_{0}^{2}}{q_{1}} \mathcal{W}_{a}+\omega_{a}^{*}\left\|\bar{A}_{a}\right\|_{\mathscr{V}}-\frac{\left(\omega_{a}^{*}\right)^{2}}{\theta_{a}}\left\|\bar{A}_{a}\right\|_{\mathscr{V}}^{2} .
\end{gathered}
$$

Let us now define the next function $\Psi^{*}=\omega_{a}^{*}\left\|\bar{A}_{a}\right\|_{\mathscr{V}}-\frac{\left(\omega_{a}^{*}\right)^{2}}{\theta_{a}}\left\|\bar{A}_{a}\right\|_{\mathscr{V}}^{2}$. Since $\Psi^{*}$ has a maximum value of $\Psi_{m}^{*}=\frac{\theta_{a}}{4}$ at $\omega_{a}^{*}\left\|\bar{A}_{a}\right\|_{\mathscr{V}}=\frac{\theta_{a}}{2}$, it follows that

$$
\frac{d \mathcal{W}_{a}}{d t} \leq-\zeta \lambda_{m} \frac{\delta_{0}^{2}}{q_{1}} \mathcal{W}_{a}+\Psi_{m}^{*} \Rightarrow \lim _{t \rightarrow \infty} \mathcal{W}_{a}(t) \leq \frac{\theta_{a} q_{1}}{4 \zeta \lambda_{m} \delta_{0}^{2}}
$$


- If $\omega_{a}\left\|\bar{A}_{a}\right\|_{\mathscr{V}}<\theta_{a}$ and $\omega_{a}^{*}\left\|\bar{A}_{a}\right\|_{\mathscr{V}} \geq \theta_{a}$ then

$$
\begin{gathered}
\frac{d \mathcal{W}_{a}}{d t} \leq-\zeta \lambda_{m} \frac{\delta_{0}^{2}}{q_{1}} \mathcal{W}_{a}+\lambda_{q}\left\|\gamma_{d a}\right\|_{2}\left\|\bar{A}_{a}\right\|_{\mathscr{V}}+\eta_{a}\left\|\bar{A}_{a}\right\|_{\mathscr{V}}-\frac{\left(\omega_{a}\right)^{2}}{\theta_{a}}\left\|\bar{A}_{a}\right\|_{\mathscr{V}}^{2}-\omega_{a}^{*}\left\|\bar{A}_{a}\right\|_{\mathscr{V}} \leq \\
-\zeta \lambda_{m} \frac{\delta_{0}^{2}}{q_{1}} \mathcal{W}_{a}+\omega_{a}\left\|\bar{A}_{a}\right\|_{\mathscr{V}}-\frac{\omega_{a}^{2}}{\theta_{a}}\left\|\bar{A}_{a}\right\|_{\mathscr{V}}^{2} .
\end{gathered}
$$

Using the same arguments employed in the previous point, we obtain:

$$
\frac{d \mathcal{W}_{a}}{d t} \leq-\zeta \lambda_{m} \frac{\delta_{0}^{2}}{q_{1}} \mathcal{W}_{a}+\Psi_{m} \Rightarrow \lim _{t \rightarrow \infty} \mathcal{W}_{a}(t) \leq \frac{\theta_{a} q_{1}}{4 \zeta \lambda_{m} \delta_{0}^{2}}
$$

where $\Psi_{m}=\frac{\theta_{a}}{4}$ is the maximum of the function $\Psi=\omega_{a}\left\|\bar{A}_{a}\right\|_{\mathscr{V}}-\frac{\omega_{a}^{2}}{\theta_{a}}\left\|\bar{A}_{a}\right\|_{\mathscr{V}}^{2}$.

- If $\omega_{a}\left\|\bar{A}_{a}\right\|_{\mathscr{V}}<\theta_{a}$ and $\omega_{a}^{*}\left\|\bar{A}_{a}\right\|_{\mathscr{V}}<\theta_{a}$ then

$$
\begin{gathered}
\frac{d \mathcal{W}_{a}}{d t} \leq-\zeta \lambda_{m} \frac{\delta_{0}^{2}}{q_{1}} \mathcal{W}_{a}+\lambda_{q}\left\|\gamma_{d a}\right\|_{2}\left\|\bar{A}_{a}\right\|_{\mathscr{V}}+\eta_{a}\left\|\bar{A}_{a}\right\|_{\mathscr{V}}-\frac{\left(\omega_{a}\right)^{2}}{\theta_{a}}\left\|\bar{A}_{a}\right\|_{\mathscr{V}}^{2}- \\
\frac{\left(\omega_{a}^{*}\right)^{2}}{\theta_{a}}\left\|\bar{A}_{a}\right\|_{\mathscr{V}}^{2} \leq-\zeta \lambda_{m} \frac{\delta_{0}^{2}}{q_{1}} \mathcal{W}_{a}+\omega_{a}\left\|\bar{A}_{a}\right\|_{\mathscr{V}}+\omega_{a}^{*}\left\|\bar{A}_{a}\right\|_{\mathscr{V}}-\frac{\left(\omega_{a}\right)^{2}}{\theta_{a}}\left\|\bar{A}_{a}\right\|_{\mathscr{V}}^{2}- \\
\frac{\left(\omega_{a}^{*}\right)^{2}}{\theta_{a}}\left\|\bar{A}_{a}\right\|_{\mathscr{V}}^{2} .
\end{gathered}
$$

Making use of functions $\Psi$ and $\Psi^{*}$ defined in the previous points, it is easy to see that

$$
\frac{d \mathcal{W}_{a}}{d t} \leq-\zeta \lambda_{m} \frac{\delta_{0}^{2}}{q_{1}} \mathcal{W}_{a}+\frac{\theta_{a}}{2} \Rightarrow \lim _{t \rightarrow \infty} \mathcal{W}_{a}(t)=\frac{\theta_{a} q_{1}}{2 \zeta \lambda_{m} \delta_{0}^{2}}
$$

which implies that $\mathcal{W}_{a}$, and then $\bar{z}_{a}$, are ultimately bounded.

\section{References}

Alonso, A., Banga, J., Moles, C., Balsa-Canto, E., 2002a. Identification and control design numbers in non-linear diffusion-convection reaction processes. In: Proceedings of the 10th Mediterranean Conference on Control and $\mathrm{Au}-$ tomation - MED2002, Lisbon, Portugal.

Alonso, A., Ydstie, B., Banga, J., 2002b. From irreversible thermodynamics to a robust control theory for distributed process systems. Journal of Process Control 12 (4), 507-517.

Alonso, A. A., Banga, J. R., Sánchez, I., 2000. Passive control design for distributed process systems: Theory and applications. AIChE Journal 46 (8), $1593-1606$.

Alonso, A. A., Fernández, C. V., Banga, J. R., 2004a. Dissipative systems: from physics to robust nonlinear control. Int. J. Robust Nonlinear Control $14(2), 157-179$. 
Alonso, A. A., Frouzakis, C. E., Kevrekidis, I. G., 2004b. Optimal sensor placement for state reconstruction of distributed process systems. AIChE Journal 50 (7), 1438-1452.

Alonso, A. A., Kevrekidis, I., Balsa-Canto, E., Banga, J. R., 2002c. Robust nonlinear control design of distributed process systems with input constraints. In: Proceedings of (IFAC 2002). Barcelona, Spain.

Alonso, A. A., Ydstie, B. E., 2001. Stabilization of distributed systems using irreversible thermodynamics. Automatica 37 (11), 1739-1755.

Antoniades, C., Christofides, P. D., 2000. Computation of optimal actuator locations for nonlinear controllers in transport reaction processes. Comp. Chem. Eng. 24 (2-7), 577-583.

Antoniades, C., Christofides, P. D., 2001. Studies on nonlinear dynamics and control of a tubular reactor with recycle. Nonlinear Analysis 47 (9), 59335944 .

Antoniades, C., Christofides, P. D., 2002. Integrated optimal actuator/sensor placement and robust control of uncertain transport-reaction processes. Computers \& Chemical Engineering 26 (2), 187-203.

Balsa-Canto, E., Alonso, A. A., Banga, J. R., 2004. Reduced-order models for nonlinear distributed process systems and their application in dynamic optimization. Industrial \& Engineering Chemistry Research 43 (13), 33533363.

Callen, H., 1985. Thermodynamics and an Introduction to Thermostatistics. 2nd. Edition. John Wiley \& Sons.

Christofides, P. D., 2001. Nonlinear and Robust Control of PDE Systems: Methods and Applications to Transport-Reaction Processes. Birkhäuser, Boston.

Christofides, P. D., Daoutidis, P., 1996. Nonlinear control of diffusionconvection-reaction processes. Comp. Chem. Eng. 20, s1071-s1076.

Conde, J. C., González, P., Lusquios, F., Chiussi, S., Serra, J., León, B., 2005. Analytical and numerical calculations of the temperature distribution in si and ge targets irradiated by excimer lasers. Applied Surface Science 248 (14), 455-460.

Demetriou, M., Kazantzis, N., 2005. A new integrated output feedback controller synthesis and collocated actuator/sensor scheduling framework for distributed parameter processes. Computers and Chemical Engineering 29 (4), 867-876.

Dennis Jr., J. E., Schnabel, R. B., 1983. Numerical Methods for Unconstrained Optimization and Nonlinear Equations. Prentice Hall.

Dochain, D., Babary, J., Tali-Maamar, N., 1992. Modelling and adaptive control of nonlinear distributed parameter bioreactors via orthogonal collocation. Automatica 28 (5), 873-883.

Gundepudi, P., Friedly, J., 1998. Velocity control of hyperbolic partial differential equation systems with single characteristic variable. Chem. Eng. Sci. 53 (24), 4055-4072.

Gustafson, K., 1987. Introduction to Partial Differential Equations and Hilbert 
Space Methods, 2nd Edition. John Wiley \& Sons.

Holmes, P. J., Lumley, J. L., Berkooz, G., Mattingly, J. C., Wittenberg, R. W., 1997. Low-dimensional models of coherent structures in turbulence. Physics Reports 287 (4), 337-384.

Hoo, K., Zheng, D., 2001. Low-order control-relevant models for a class of distributed parameter systems. Chemical Engineering Science 56 (23), 66836710 .

Khalil, H. K., 1996. Nonlinear Systems, 2nd Edition. Prentice Hall, Upper Saddle River, New Jersey.

Reddy, B., 1998. Introductory Functional Analysis: With Applications to Boundary Value Problems and Finite Elements. Springer-Verlag, New York.

Saro, O., Nonino, C., Comini, G., 1995. An enthalpy-based algorithm for the analysis of phase change in nonhomogeneous media. Computational Mechanics Publications, Southampton, pp. 127-134.

Scheerlinck, N., Verboven, P., Fikiin, K., De Baerdemaeker, J., Nicolai, B., 2001. Finite element computation of unsteady phase change heat transfer during freezing or thawing of food using a combined enthalpy and kirchhoff transform method. Transactions of the ASAE 44 (2), 429-438.

Shi, D., El-Farra, N. H., Li, M. H., Mhaskar, P., Christofides, P. D., 2006. Predictive control of particle size distribution in particulate processes. Chemical Engineering Science 61 (1), 268-281.

Shvartsman, S. Y., Kevrekidis, I. G., 1998. Nonlinear model reduction for control of distributed systems: A computer-assisted study. AIChE Journal 44 (7), 1579-1595.

Sirovich, L., 1987. Turbulence and the dynamics of coherent structures. Part I: Coherent structures. Quaterly of Appl. Math. 45 (3), 561-571.

Smagina, Y., Nekhamkina, O., Sheintuch, M., 2002. Stabilization of fronts in a reaction - diffusion system: Application of the gershgorin theorem. Ind. Eng. Chem. Res. 41 (8), 2023-2032.

Smith, J. M., Van Ness, H. C., Abbott, M. M., 1996. Introduction to Chemical Engineering Thermodynamics, 5th Edition. McGraw-Hill, New York.

Smoller, J., 1994. Shock Waves and Reaction-Diffusion Equations, 2nd Edition. Springer-Verlag, New york.

Vilas, C., García, M. R., Banga, J. R., Alonso, A. A., 2006. Stabilization of inhomogeneous patterns in a diffusion-reaction system under structural and parametric uncertainties. Journal of Theoretical Biology 241 (2), 295-306.

Ydstie, B. E., Alonso, A. A., 1997. Process systems and passivity via the clausius-plank inequality. Syst. \& Cont. Letters 30 (5), 253-264. 